\title{
Robust parameter estimations using L-moments, TL-moments and the order statistics
}

\author{
Diana Bílková ${ }^{1,2}$ \\ ${ }^{1}$ Department of Statistics and Probability, Faculty of Informatics and Statistics, University of Economics, Prague, Czech Republic \\ ${ }^{2}$ Department of Informatics and Mathematics, Faculty of Economic Studies, University of Finance and Administration, Czech Republic
}

\section{Email address:}

bilkova@vse.cz,diana.bilkova@vsfs.cz

\section{To cite this article:}

Diana Bílková. Robust Parameter Estimations Using L-Moments, TL-Moments and the Order Statistics. American Journal of Applied Mathematics. Vol. 2, No. 2, 2014, pp. 36-53. doi: 10.11648/j.ajam.20140202.11

\begin{abstract}
Application of the method of moments for the parametric distribution is common in the construction of a suitable parametric distribution. However, moment method of parameter estimation does not produce good results. An alternative approach when constructing an appropriate parametric distribution for the considered data file is to use the so-called order statistics. This paper deals with the use of order statistics as the methods of L-moments and TL-moments of parameter estimation. L-moments have some theoretical advantages over conventional moments. L-moments have been introduced as a robust alternative to classical moments of probability distributions. However, L-moments and their estimations lack some robust features that belong to the TL-moments. TL-moments represent an alternative robust version of L-moments, which are called trimmed L-moments. This paper deals with the use of L-moments and TL-moments in the construction of models of wage distribution. Three-parametric lognormal curves represent the basic theoretical distribution whose parameters were simultaneously estimated by three methods of point parameter estimation and accuracy of these methods was then evaluated. There are method of TL-moments, method of L-moments and maximum likelihood method in combination with Cohen's method. A total of 328 wage distribution has been the subject of research.
\end{abstract}

Keywords: Order Statistics, L-Moments, Tl-Moments, Maximum Likelihood Method, Probability Density Function, Distribution Function, Quantile Function, Lognormal Curves, Model of Wage Distribution

\section{Introduction}

Moments and cumulants are traditionally used to characterize the probability distribution or the observed data set in statistics. It is sometimes difficult to determine exactly what information about the shape of the distribution is expressed by its moments of third and higher order. Especially in the case of a small sample, numerical values of sample moments can be very different from the values of theoretical moments of the probability distribution from which the random sample comes. Particularly in the case of small samples, parameter estimations of the probability distribution obtained using the moment method are often markedly less accurate than estimates obtained using other methods, such as maximum likelihood method.

\section{L-Moments}

An alternative approach is to use the order statistics. Let $X$ be a random variable having a distribution with distribution function $F(x)$ and with quantile function $x(F)$, and let $X_{1}$, $X_{2}, \ldots, X n$ is a random sample of sample size $\mathrm{n}$ from this distribution. Then $X_{1: n} \leq X_{2: n} \leq \ldots \leq X_{n: n}$ are the order statistics of random sample of sample size n, which comes from the distribution of random variable $X$.

L-moments are analogous to conventional moments and are estimated based on linear combinations of order statistics, i.e. L-statistics. L-moments are an alternative system describing the shape of the probability distribution.

L-moments present the basis for a general theory, which includes the characterization and description of the theoretical probability distribution, characterization and description of the obtained sample data sets, parameter estimation of theoretical probability distribution and hypothesis testing of parameter values for the theoretical probability distribution. The theory of L-moments includes such established procedures such as the use of order statistics and Gini's middle difference and leads to some promising innovations in the area of measuring skewness and kurtosis of the distribution and provides relatively new 
methods of parameter estimation for individual distribution. L-moments can be defined for any random variable whose expected value exists. The main advantage of the L-moments than conventional moments consists in the fact that L-moments can be estimated on the basis of linear functions of the data and are more resistant to the influence of sample variation. Compared to conventional moments, L-moments are more robust to the existence of outliers in the data and allow better conclusions obtained on the basis of small samples for basic probability distribution. L-moments often bring even more efficient parameter estimations of parametric distribution than the estimations obtained using maximum likelihood method, especially for small samples. Theoretical advantages of L-moments over conventional moments lie in the ability to characterize a wider range of distribution and in greater resistance to the presence of outliers in the data when estimating from the sample. Compared with conventional moments, experience also shows that L-moments are less prone to bias estimation and approximation by asymptotic normal distribution is more accurate in finite samples.

\subsection{L-Moments of Probability Distribution}

Let $\mathrm{X}$ be a continuous random variable that has a distribution with distribution function $F(x)$ and with quantile function $x(F)$. Let $X_{1: n} \leq X_{2: n} \leq \ldots \leq X_{n: n}$ are the order statistics of random sample of sample size $n$, which comes from the distribution of random variable $X$. L-moment of the $r$-th order of random variable $X$ is defined

$$
\lambda_{r}=\frac{1}{r} \cdot \sum_{j=0}^{r-1}(-1)^{j} \cdot\left(\begin{array}{c}
r-1 \\
j
\end{array}\right) \cdot E\left(X_{r-j: r}\right), \quad r=1,2, \ldots .
$$

Expected value of the $r$-th order statistic of random sample of sample size $n$ has the form

$$
E\left(X_{r: n}\right)=\frac{n !}{(r-1) ! \cdot(n-r) !} \cdot \int_{0}^{1} x(F) \cdot[F(x)]^{r-1} \cdot[1-F(x)]^{n-r} \mathrm{~d} F(x) .
$$

If we substitute equation (2) into equation (1), we obtain after adjustments

$$
\lambda_{r}=\int_{0}^{1} x(F) \cdot P_{r-1}^{*}[F(x)] \mathrm{d} F(x), \quad r=1,2, \ldots,
$$

where

$$
P_{r}^{*}[F(x)]=\sum_{j=0}^{r} p_{r, j}^{*} \cdot[F(x)]^{j} \quad \text { a } \quad p_{r, j}^{*}=(-1)^{r-j} \cdot\left(\begin{array}{c}
r \\
j
\end{array}\right) \cdot\left(\begin{array}{c}
r+j \\
j
\end{array}\right),
$$

and $P_{r}^{*}[F(x)]$ represents the $r$-th shifted Legendre polynomial. We also obtain substituting (2) into equation (1)

$\lambda_{r}=\frac{1}{r} \cdot \sum_{j=0}^{r-1}(-1)^{j} \cdot\left(\begin{array}{c}r-1 \\ j\end{array}\right) \cdot \frac{r !}{(r-j-1) ! \cdot j !} \cdot \int_{0}^{1} x(F) \cdot[F(x)]^{r-j-1} \cdot[1-F(x)]^{j} \mathrm{~d} F(x), \quad r=1,2, \ldots$

The letter " $L$ " in the name of "L-moments" stresses that the $r$-th L-moment $\lambda_{r}$ is a linear function of the expected value of certain linear combination of order statistics. Own estimation of the $r$-th L-moment $\lambda_{r}$ based on the obtained data sample is then linear combination of ordered sample values, i.e. L-statistics. The first four L-moments of the probability distribution in now defined

$$
\begin{gathered}
\lambda_{1}=E\left(X_{1: 1}\right)=\int_{0}^{1} x(F) \mathrm{d} F(x), \\
\lambda_{2}=\frac{1}{2} E\left(X_{2: 2}-X_{1: 2}\right)=\int_{0}^{1} x(F) \cdot[2 F(x)-1] \mathrm{d} F(x) \\
\lambda_{3}=\frac{1}{3} E\left(X_{3: 3}-2 X_{2: 3}+X_{1: 3}\right)=\int_{0}^{1} x(F) \cdot\left\{6[F(x)]^{2}-6 F(x)+1\right\} \mathrm{d} F(x) \\
\lambda_{4}=\frac{1}{4} E\left(X_{4: 4}-3 X_{3: 4}+3 X_{2: 4}-X_{1: 4}\right)=\int_{0}^{1} x(F) \cdot\left\{20[F(x)]^{3}-30[F(x)]^{2}+12[F(x)]-1\right\} \mathrm{d} F(x) .
\end{gathered}
$$

The probability distribution can be specified by its L-moments, even if some its conventional moments do not exist, but the opposite is not true. It can be proved that the first L-moment $\lambda_{1}$ is the level characteristic of the probability distribution, the second L-moment $\lambda_{2}$ is the variability characteristic, of a random variable $X$. It is convenient to standardize the higher L-moments $\lambda_{r}, r \geq 3$, to be independent on specific units of the random variable $X$. The ratio of L-moments of the $r$-th order of random variable $X$ is defined

$$
\tau_{r}=\frac{\lambda_{r}}{\lambda_{2}}, \quad r=3,4, \ldots
$$

It is also possible to define such a function of L-moments, which is analogous to the classical coefficient of variation, i.e. the so-called L-coefficient of variation

$$
\tau=\frac{\lambda_{2}}{\lambda_{1}} .
$$

The ratio of L-moments $\tau_{3}$ is the skewness characteristic and the ratio of L-moments $\tau_{4}$ is the kurtosis characteristic of the corresponding probability distribution. Main properties of the probability distribution are summarized very well by the following four characteristics: L-location $\lambda_{1}$, L-variation $\lambda_{2}$, L-skewness $\tau_{3}$ and L-kurtosis $\tau_{4}$. L-moments $\lambda_{1}$ and $\lambda_{2}$, L-coefficient of variation $\tau$ and ratios of L-moments $\tau_{3}$ and $\tau_{4}$ are the most useful measurements for characterizing the probability distribution. Their most important features are: the existence (if the expected value of the distribution exists, then all L-moments of the distribution exit, too) and uniqueness (if the expected value of the distribution exists, then L-moments define only one distribution, i.e. no two distributions have the same L-moments).

Using equations (6)-(8) and equation (10) we obtain formulas for L-moments, respectively for the ratios of L-moments for the case of chosen probability distributions, see Table 1. More on the L-moments is for example in [1], [2] and [3]. 
Table 1. Formulas for distribution or quantile functions, L-moments and their ratios for chosen probability distributions.

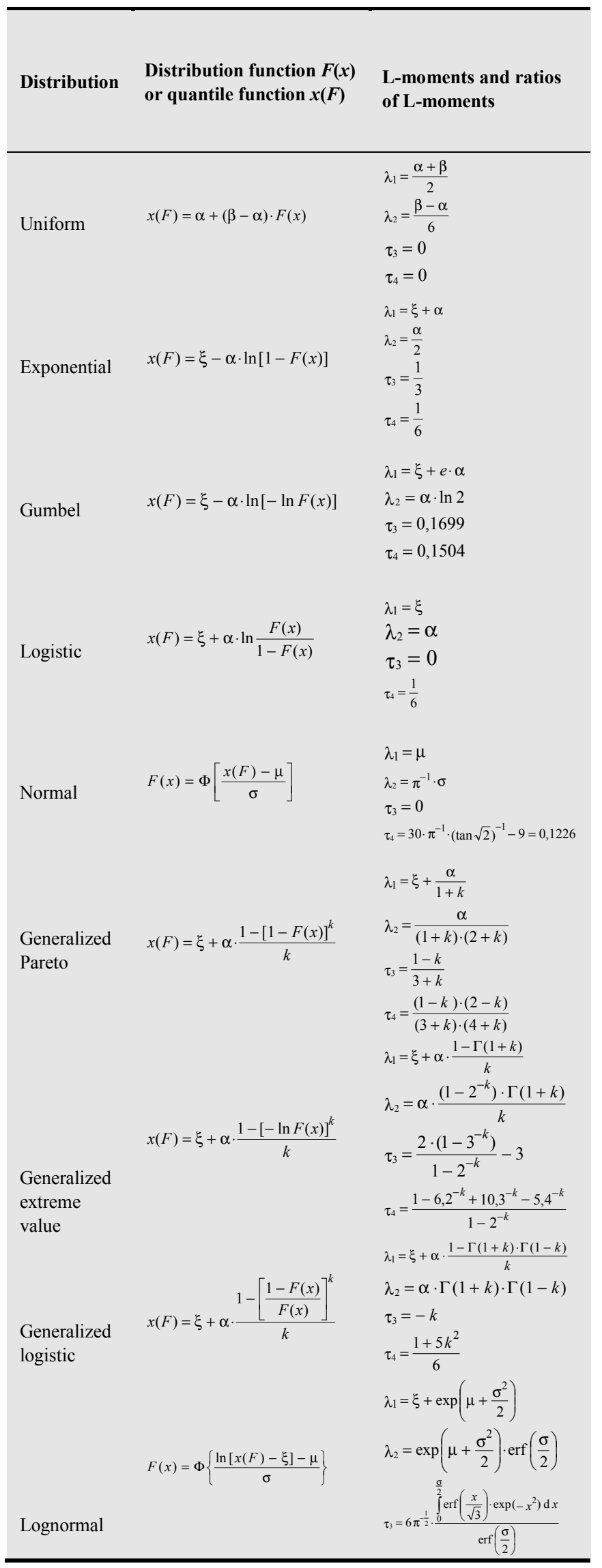

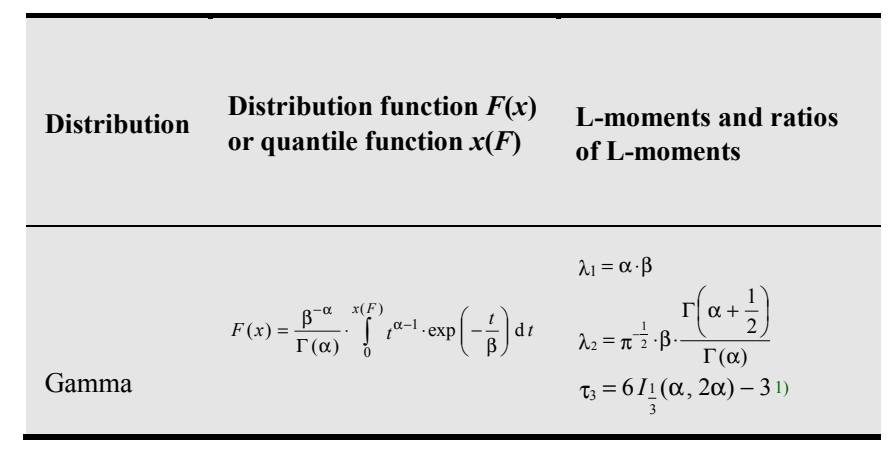

Source: [1]; own research

\subsection{Sample L-Moments}

We usually estimate L-moments using random sample, which is taken from an unknown distribution. Since the r-th L-moment $\lambda \mathrm{r}$ is a function of the expected values of order statistics of random sample of sample size $r$, it is natural to estimate it using the so-called U-statistic, i.e. the corresponding function of sample order statistics (averaged over partial subsets of sample size $r$, which can be formed from the obtained random sample of sample size $n$ ).

Let $x_{1}, x_{2}, \ldots, x_{n}$ is a sample and $x_{1: n} \leq x_{2: n} \leq \ldots \leq x_{n: n}$ is an ordered sample. Then the $r$-th sample L-moment can be written as

$$
l_{r}=\left(\begin{array}{l}
n \\
r
\end{array}\right)^{-1} \sum_{1 \leq i 1<i 2<\ldots<i r \leq n} \sum_{i} \frac{1}{r} \cdot \sum_{j=0}^{r-1}(-1)^{j} \cdot\left(\begin{array}{c}
r-1 \\
j
\end{array}\right) \cdot x_{i r-j: n}, \quad r=1,2, \ldots, n .
$$

Hence the first four sample L-moments have the form

$$
\begin{gathered}
l_{1}=\frac{1}{n} \cdot \sum_{i} x_{i}, \\
l_{2}=\frac{1}{2} \cdot\left(\begin{array}{l}
n \\
2
\end{array}\right)^{-1} \cdot \sum_{i>j}\left(x_{i: n}-x_{j: n}\right), \\
l_{3}=\frac{1}{3} \cdot\left(\begin{array}{l}
n \\
3
\end{array}\right)^{-1} \cdot \sum_{i>j>k}\left(x_{i: n}-2 x_{j: n}+x_{k: n}\right), \\
l_{4}=\frac{1}{4} \cdot\left(\begin{array}{l}
n \\
4
\end{array}\right)^{-1} \cdot \sum_{i>j>k>l}\left(x_{i: n}-3 x_{j: n}+3 x_{k: n}-x_{l: n}\right) .
\end{gathered}
$$

U-statistics are widely used especially in nonparametric statistics. Their positive features are: the absence of bias, asymptotic normality and some slight resistance due to the influence of outliers.

When calculating the $r$-th sample L-moment it is not necessary to repeat the calculation across all partial subsets of sample size $r$, but this statistic can be expressed directly as linear combination of order statistics of random sample of sample size $n$. If we consider the estimation of $E\left(X_{r: r}\right)$, which is taken using U-statistics, this estimate can be written as $r \cdot b_{r-1}$, where

\footnotetext{
${ }^{1)} I_{x}(p, q)$ is incomplete beta function
} 


$$
b_{r}=\frac{1}{n} \cdot\left(\begin{array}{c}
n-1 \\
r
\end{array}\right)^{-1} \cdot \sum_{j=r+1}^{n}\left(\begin{array}{c}
j-1 \\
r
\end{array}\right) \cdot x_{j: n},
$$

specifically

$$
\begin{gathered}
b_{0}=\frac{1}{n} \cdot \sum_{j=1}^{n} x_{j: n}, \\
b_{1}=\frac{1}{n} \cdot \sum_{j=2}^{n} \frac{(j-1)}{(n-1)} \cdot x_{j: n}, \\
b_{2}=\frac{1}{n} \cdot \sum_{j=3}^{n} \frac{(j-1) \cdot(j-2)}{(n-1) \cdot(n-2)} \cdot x_{j: n},
\end{gathered}
$$

therefore generally

$$
b_{r}=\frac{1}{n} \cdot \sum_{j=r+1}^{n} \frac{(j-1) \cdot(j-2) \cdot \ldots \cdot(j-r)}{(n-1) \cdot(n-2) \cdot \ldots \cdot(n-r)} \cdot x_{j: n} .
$$

Therefore the first four sample L-moments can be written as

$$
\begin{gathered}
l_{1}=b_{0}, \\
l_{2}=2 b_{1}-b_{0}, \\
l_{3}=6 b_{2}-6 b_{1}+b_{0}, \\
l_{4}=20 b_{3}-30 b_{2}+12 b_{1}-b_{0} .
\end{gathered}
$$

Thus, we can write universally

$$
l_{r+1}=\sum_{k=0}^{r} p_{r, k}^{*} b_{k}, \quad r=0,1, \ldots, n-1,
$$

where

$$
p_{r, k}^{*}=(-1)^{r-k} \cdot\left(\begin{array}{l}
r \\
k
\end{array}\right) \cdot\left(\begin{array}{c}
r+k \\
k
\end{array}\right)=\frac{(-1)^{r-k} \cdot(r+k) !}{(k !)^{2} \cdot(r-k) !} .
$$

Application of sample L-moments is similar to the application of sample conventional moments. Sample L-moments summarize the basic properties of the sample distribution, which are the location (level), variability, skewness and kurtosis. Thus, sample L-moments estimate the corresponding properties of the probability distribution from which the sample comes and can be used in estimating the parameters of the relevant theoretical probability distribution. Under such applications, we often prefer the L-moments before conventional moments, since as a linear function of data, sample L-moments are less sensitive to a sample variability than conventional moments or to the size of errors in the case of existence of outliers. L-moments therefore lead to more accurate and robust estimations of the parameters or characteristics of a basic probability distribution, see for example [4]-[12].

Sample L-moments were used already previously in the statistics, although not as a part of a unified theory. The first sample L-moment $l_{1}$ is a sample L-location (sample average), the second sample L-moment $l_{2}$ is a sample L-variability. Natural estimation of the ratio of L-moments (10) is the sample ratio of L-moments

$$
t_{r}=\frac{l_{r}}{l_{2}}, \quad r=3,4, \ldots .
$$

Hence $t_{3}$ is a sample L-skewness and $t_{4}$ is a sample L-kurtosis. Sample ratios of L-moments $t_{3}$ and $t_{4}$ can be used as characteristics of skewness and kurtosis of the sample data file. Gini's middle difference is related to sample L-moments, which has the form

$$
G=\left(\begin{array}{l}
n \\
2
\end{array}\right)^{-1} \cdot \sum_{i>j}\left(x_{i: n}-x_{j: n}\right)
$$

and Gini's coefficient, which depends only on a single parameter $\sigma$ in the case of two-parametric lognormal distribution, but it depends on the values of all three parameters in the case of three-parametric lognormal distribution. Table 2 presents the formulas for estimation of parameters of chosen probability distributions, which were obtained using the method of L-moments.

Table 2. Formulas for parameter estimations made by the method of L-moments of chosen probability distributions.

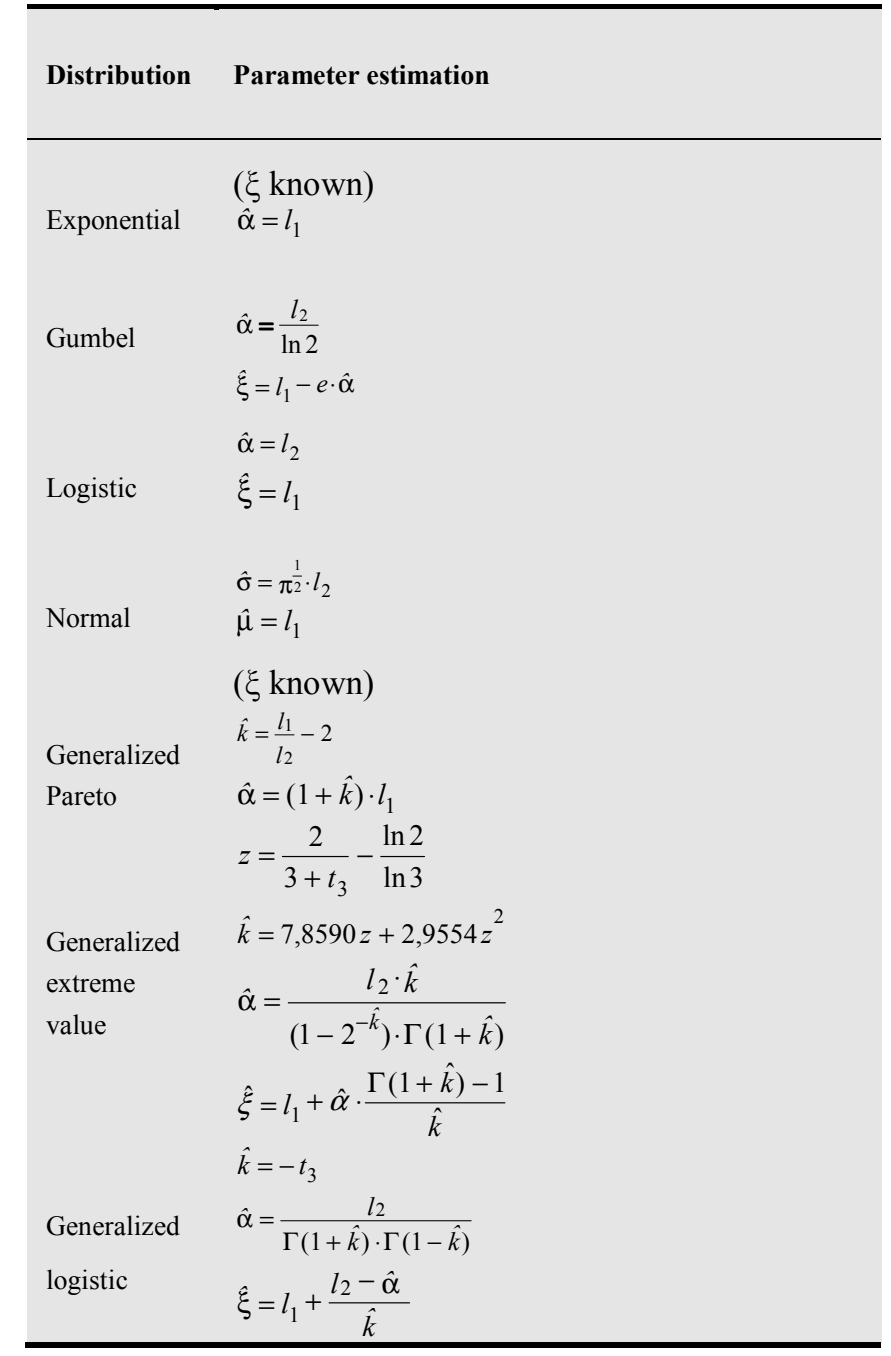




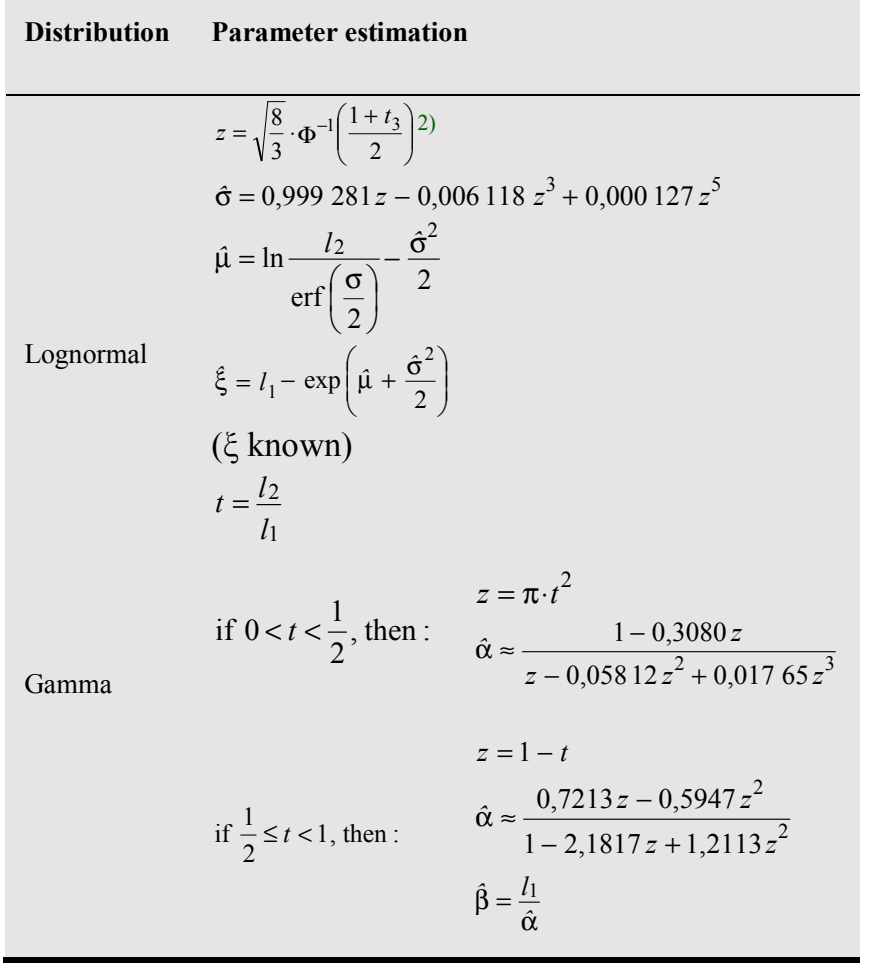

Source: [1]; own research

\section{TL-Moments}

Alternative robust version of L-moments will be now presented. This robust modification of L-moments is called „trimmed L-moments“, and labeled „TL-moments“.

This is a relatively new category of moment characteristics of the probability distribution. There are the characteristics of the level, variability, skewness and kurtosis of probability distributions constructed using TL-moments that are robust extending of L-moments. L-moments alone were introduced as a robust alternative to classical moments of probability distributions. However, L-moments and their estimations lack some robust properties that belong to the TL-moments.

Sample TL-moments are linear combinations of sample order statistics, which assign zero weight to a predetermined number of sample outliers. Sample TL-moments are unbiased estimations of the corresponding TL-moments of probability distributions. Some theoretical and practical aspects of TL-moments are still under research or remain for future research. Efficiency of TL-statistics depends on the choice of $\alpha$ proportion, for example, the first sample TL-moments $l_{1}^{(0)}, l_{1}^{(1)}, l_{1}^{(2)}$ have the smallest variance (the highest efficiency) among other estimations from random samples from normal, logistic and double exponential distribution.

When constructing the TL-moments, the expected values of order statistics of random sample in the definition of

\footnotetext{
${ }^{2)} \Phi^{-1}(\cdot)$ is a quantile function of the standardized normal distribution
}

L-moments of probability distributions are replaced by the expected values of order statistics of a larger random sample, where the sample size grows like this, so that it will correspond to the total size of modification, as shown below.

TL-moments have certain advantages over conventional L-moments and central moments. TL-moment of probability distribution may exist even if the corresponding L-moment or central moment of the probability distribution does not exist, as it is the case of Cauchy's distribution. Sample TL-moments are more resistant to existence of outliers in the data. The method of TL-moments is not intended to replace the existing robust methods, but rather as their supplement, especially in situations where we have outliers in the data.

\subsection{TL-Moments of Probability Distribution}

In this alternative robust modification of L-moments, the expected value $E\left(X_{r-j: r}\right)$ is replaced by the expected value $E\left(X_{r+t 1-j: r+t 1+t 2}\right)$. Thus, for each $r$ we increase sample size of random sample from the original $r$ to $r+t_{1}+t_{2}$ and we work only with the expected values of these $r$ treated order statistics $X_{t 1+1: r+t 1+t 2}, X_{t 1+2: r+t 1+t 2}, \ldots, X_{t 1+r: r+t 1+t 2}$ by trimming the $t_{1}$ smallest and the $t_{2}$ largest from the conceptual sample. This modification is called the $r$-th trimmed L-moment (TL-moment) and is marked $\lambda_{r}^{\left(t_{1}, t_{2}\right)}$. Thus, TL-moment of the $r$-th order of random variable $X$ is defined

$$
\lambda_{r}^{\left(t 1, t_{2}\right)}=\frac{1}{r} \cdot \sum_{j=0}^{r-1}(-1)^{j} \cdot\left(\begin{array}{c}
r-1 \\
j
\end{array}\right) \cdot E\left(X_{r+t_{1}-j: r+t_{1}+t_{2}}\right), \quad r=1,2, \ldots
$$

It is apparent from equations (30) and (1) that the TL-moments simplify to L-moments, when $t_{1}=t_{2}=0$. Although we can also consider applications, where the values of trimming are not equal, i.e. $t_{1} \neq t_{2}$, we focus here only on symmetric case $t_{1}=t_{2}=t$. Then equation (30) can be rewritten

$$
\lambda_{r}^{(t)}=\frac{1}{r} \cdot \sum_{j=0}^{r-1}(-1)^{j} \cdot\left(\begin{array}{c}
r-1 \\
j
\end{array}\right) \cdot E\left(X_{r+t-j: r+2 t}\right), \quad r=1,2, \ldots
$$

Thus, form example, $\lambda_{1}^{(t)}=E\left(X_{1+t: 1+2 t}\right)$ is the expected value of median from conceptual random sample of sample size $1+2 t$. It is necessary here to note that $\lambda_{1}^{(t)}$ is equal to zero for distributions, which are symmetrical around zero.

First four TL-moments have the form for $t=1$

$$
\begin{gathered}
\lambda_{1}^{(1)}=E\left(X_{2: 3}\right), \\
\lambda_{2}^{(1)}=\frac{1}{2} E\left(X_{3: 4}-X_{2: 4}\right), \\
\lambda_{3}^{(1)}=\frac{1}{3} E\left(X_{4: 5}-2 X_{3: 5}+X_{2: 5}\right), \\
\lambda_{4}^{(1)}=\frac{1}{4} E\left(X_{5: 6}-3 X_{4: 6}+3 X_{3: 6}-X_{2: 6}\right) .
\end{gathered}
$$

Note that the measures of location (level), variability, 
skewness and kurtosis of the probability distribution analogous to conventional L-moments (6)-(9) are based on $\lambda_{1}^{(1)}, \lambda_{2}^{(1)}, \lambda_{3}^{(1)} \mathrm{a} \lambda_{4}^{(1)}$.

Expected value $E(X r: n)$ can be written using the formula (2). Using equation (2) we can re-express the right side of equation (31)

$\lambda_{r}^{(t)}=\frac{1}{r} \cdot \sum_{j=0}^{r-1}(-1)^{j} \cdot\left(\begin{array}{c}r-1 \\ j\end{array}\right) \cdot \frac{(r+2 t) !}{(r+t-j-1) ! \cdot(t+j) !} \cdot \int_{0}^{1} x(F) \cdot[F(x)]^{r+t-j-1} \cdot[1-F(x)]^{t+j} \mathrm{~d} F(x), r=1,2, \ldots$.

It is necessary to be noted here that $\lambda_{r}^{(0)}=\lambda_{r}$ is a normal the r-th L-moment without any trimming.

Expressions (32)-(35) for the first four TL-moments, where $t=1$, can be written in an alternative manner

$$
\begin{gathered}
\lambda_{1}^{(1)}=6 \cdot \int_{0}^{1} x(F) \cdot[F(x)] \cdot[1-F(x)] \mathrm{d} F(x), \\
\lambda_{2}^{(1)}=6 \cdot \int_{0}^{1} x(F) \cdot[F(x)] \cdot[1-F(x)] \cdot[2 F(x)-1] \mathrm{d} F(x), \\
\lambda_{3}^{(1)}=\frac{20}{3} \cdot \int_{0}^{1} x(F) \cdot[F(x)] \cdot[1-F(x)] \cdot\left\{5[F(x)]^{2}-5 F(x)+1\right\} \mathrm{d} F(x) \\
\lambda_{4}^{(1)}=\frac{15}{2} \cdot \int_{0}^{1} x(F) \cdot[F(x)] \cdot[1-F(x)] \cdot\left\{14[F(x)]^{3}-21[F(x)]^{2}+9[F(x)]-1\right] \mathrm{d} F(x) .
\end{gathered}
$$

Distribution may be identified by its TL-moments, although some of its L-moments or conventional central moments do not exit; for example $\lambda_{1}^{(1)}$ (expected value of median of conceptual random sample of sample size three) exists for Cauchy's distribution, although the first L-moment $\lambda 1$ does not exist.

TL-skewness $\tau_{3}^{(t)}$ and TL-kurtosis $\tau_{4}^{(t)}$ are defined analogously as L-skewness $\tau_{3}$ and L-kurtosis $\tau_{4}$

$$
\begin{gathered}
\tau_{3}^{(t)}=\frac{\lambda_{3}^{(t)}}{\lambda_{2}^{(t)}}, \\
\tau_{4}^{(t)}=\frac{\lambda_{4}^{(t)}}{\lambda_{2}^{(t)}} .
\end{gathered}
$$

\subsection{Sample TL-Moments}

Let $x_{1}, x_{2}, \ldots, x_{n}$ is a sample and $x_{1: n} \leq x_{2: n} \leq \ldots \leq x_{n: n}$ is an ordered sample. Expression

$$
\hat{E}\left(X_{j+1: j+l+1}\right)=\frac{1}{\left(\begin{array}{c}
n \\
j+l+1
\end{array}\right)} \cdot \sum_{i=1}^{n}\left(\begin{array}{c}
i-1 \\
j
\end{array}\right) \cdot\left(\begin{array}{c}
n-i \\
l
\end{array}\right) \cdot x_{i: n}
$$

is considered to be an unbiased estimation of expected value of the $(j+1)$-th order statistic $X_{j+1: j+l+1}$ in conceptual random sample of sample size $(j+l+1)$. Now we will assume that we replace the expression $E\left(X_{r+t-j: r+2 t}\right)$ by its unbiased estimation in the definition of the $r$-th TL-moment $\lambda_{r}^{(t)}$ in (31)

$$
\hat{E}\left(X_{r+t-j \cdot r+2 t}\right)=\frac{1}{\left(\begin{array}{c}
n \\
r+2 t
\end{array}\right)} \cdot \sum_{i=1}^{n}\left(\begin{array}{c}
i-1 \\
r+t-j-1
\end{array}\right) \cdot\left(\begin{array}{c}
n-i \\
t+j
\end{array}\right) \cdot x_{i: n},
$$

which we gain by assigning $j \rightarrow r+t-j-1$ a $l \rightarrow t+j$ in (43). Now we obtain the $r$-th sample TL-moment

$$
l_{r}^{(t)}=\frac{1}{r} \cdot \sum_{j=0}^{r-1}(-1)^{j} \cdot\left(\begin{array}{c}
r-1 \\
j
\end{array}\right) \cdot \hat{E}\left(X_{r+t-j: r+2 t}\right), \quad r=1,2, \ldots, n-2 t,
$$

i.e.

$$
l_{r}^{(t)}=\frac{1}{r} \cdot \sum_{j=0}^{r-1}(-1)^{j} \cdot\left(\begin{array}{c}
r-1 \\
j
\end{array}\right) \cdot \frac{1}{\left(\begin{array}{c}
n \\
r+2 t
\end{array}\right)} \cdot \sum_{i=1}^{n}\left(\begin{array}{c}
i-1 \\
r+t-j-1
\end{array}\right) \cdot\left(\begin{array}{c}
n-i \\
t+j
\end{array}\right) \cdot x_{i: n}, \quad r=1,2, \ldots, n-2 t,
$$

which is unbiased estimation of the $r$-th TL-moment $\lambda_{r}^{(t)}$. Note that for each $j=0,1, \ldots, r-1$, values $x_{i: n}$ in (46) are nonzero only for $r+t-j \leq i \leq n-t-j$ due to the combinatorial numbers. Simple adjustment of the equation (46) provides an alternative linear form

$$
l_{r}^{(t)}=\frac{1}{r} \cdot \sum_{i=r+t}^{n-t}\left[\frac{\sum_{j=0}^{r-1}(-1)^{j} \cdot\left(\begin{array}{c}
r-1 \\
j
\end{array}\right)\left(\begin{array}{c}
i-1 \\
r+t-j-1
\end{array}\right) \cdot\left(\begin{array}{c}
n-i \\
t+j
\end{array}\right)}{\left(\begin{array}{c}
n \\
r+2 t
\end{array}\right)}\right] \cdot x_{i: n} .
$$

For example, we obtain for $r=1$ for the first sample TL-moment

$$
l_{1}^{(t)}=\sum_{i=t+1}^{n-t} w_{i: n}^{(t)} \cdot x_{i: n}
$$

where the weights are given by

$$
w_{i: n}^{(t)}=\frac{\left(\begin{array}{c}
i-1 \\
t
\end{array}\right) \cdot\left(\begin{array}{c}
n-i \\
t
\end{array}\right)}{\left(\begin{array}{c}
n \\
2 t+1
\end{array}\right)} .
$$

The above results can be used to estimate TL-skewness and TL-kurtosis by simple ratios

$$
t_{3}^{(t)}=\frac{l_{3}^{(t)}}{l_{2}^{(t)}}
$$

$$
t_{4}^{(t)}=\frac{l_{4}^{(t)}}{l_{2}^{(t)}}
$$

We can choose $t=n \alpha$ representing the amount of the adjustment from each end of the sample, where $\alpha$ is a certain proportion, where $0 \leq \alpha<0,5$.

Table 3 contains the formulas for TL-moments and for the ratios of TL-moments and the formulas for parameter estimations obtained using the method of TL-moments of chosen probability distributions. More on the TL-moments is for example in [13]. 
Table 3. Formulas for TL-moments and their ratios and parameter estimations made by the method of TL-moments of the chosen probability distributions $(t=1)$.

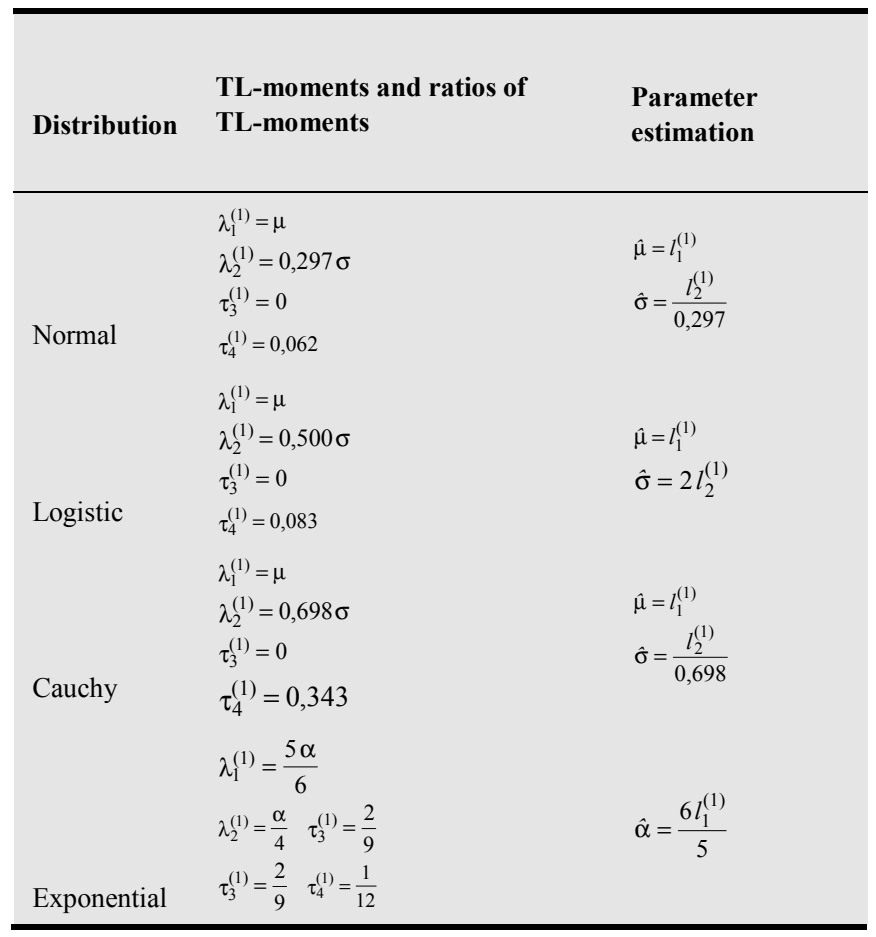

Source: [13]; own research

\section{Lognormal Curves}

\subsection{Three-Parametric Lognormal Curves}

Random variable $X$ has three-parametric lognormal distribution with parameters $\mu, \sigma^{2}$ and $\theta$, where $-\infty<\mu<\infty$, $\sigma^{2}>0,-\infty<\theta<\infty$, if its probability density function have the form

$$
\begin{aligned}
f\left(x ; \mu, \sigma^{2}, \theta\right) & =\frac{1}{\sigma \cdot(x-\theta) \cdot \sqrt{2 \pi}} \cdot \exp \left[-\frac{[\ln (x-\theta)-\mu]^{2}}{2 \sigma^{2}}\right], & \\
& =0, & \text { else. }
\end{aligned}
$$

Lognormal distribution with parameters $\mu, \sigma^{2}$ and $\theta$ (beginning of distribution, theoretical minimum) is marked $\operatorname{LN}\left(\mu, \sigma^{2}, \theta\right)$. Probability density function of three-parametric lognormal distribution is asymmetric, positively skewed. Figures 1 and 2 show the graphs of the probability density function of three-parametric lognormal distribution depending on the values of the parameters of this distribution.

Probability density function of three-parametric lognormal distribution is sometimes presented in the form

$$
f(x ; \gamma, \delta, \theta) \quad=\frac{\delta}{(x-\theta) \cdot \sqrt{2 \pi}} \cdot \exp \left\{-\frac{1}{2}[\gamma+\delta \cdot \ln (x-\theta)]^{2}\right\}, \quad x>\theta,
$$

$=0$,

where it is valid $\mu=-\frac{\gamma}{\delta}$ and $\sigma=\frac{1}{\delta}$ between the expressions for probability density function (52) and (53).

If we substitute $\theta=0$ (distribution minimum) into expressions for the probability density function of three-parametric lognormal distribution (52) and (53), we obtain formulas for the probability density function of two-parametric lognormal distribution.

Distribution function of three-parametric lognormal distribution has the form

$$
F(x)=\Phi\left[\frac{\ln (x-\theta)-\mu}{\sigma}\right], \quad x>\theta .
$$

If the random variable $X$ has three-parametric lognormal distribution $\mathrm{LN}\left(\mu, \sigma^{2}, \theta\right)$, then the random variable

$$
Y=\ln (X-\theta)
$$

has normal distribution $\mathrm{N}\left(\mu, \sigma^{2}\right)$ and the random variable

$$
U=\frac{\ln (X-\theta)-\mu}{\sigma}=\gamma+\delta \cdot \ln (X-\theta)
$$

has standardized normal distribution $\mathrm{N}(0 ; 1)$. Parameter $\mu$ is the expected value of random variable (55) and parameter $\sigma^{2}$ is the variance of this random variable. Parameter $\theta$ is the beginning of the distribution, i.e. theoretical minimum of the random variable $X$.

For $\omega=\exp \left(\sigma^{2}\right)$ the $r$-th common and central moments of three-parametric lognormal distribution have the form

$$
\mu_{r}^{\prime}=E\left(X^{r}\right)=\theta+\exp \left(r \cdot \mu+\frac{r^{2} \sigma^{2}}{2}\right),
$$

$$
\mu_{r}=E\left[\left(X-\mu_{1}^{\prime}\right)^{r}\right]=\omega^{r / 2} \cdot\left[\sum_{j=0}^{r}(-1)^{j} \cdot\left(\begin{array}{l}
r \\
j
\end{array}\right) \cdot \omega^{(r-j) \cdot(r-j-1) / 2}\right] \cdot \exp (r \cdot \mu),
$$

specifically

$$
\mu_{3}=\omega^{3 / 2} \cdot(\omega-1)^{2} \cdot(\omega+2) \cdot \exp (3 \cdot \mu),
$$

$$
\mu_{4}=\omega^{2} \cdot(\omega-1)^{2} \cdot\left(\omega^{4}+2 \omega^{3}+3 \omega^{2}-3\right) \cdot \exp (4 \cdot \mu) .
$$

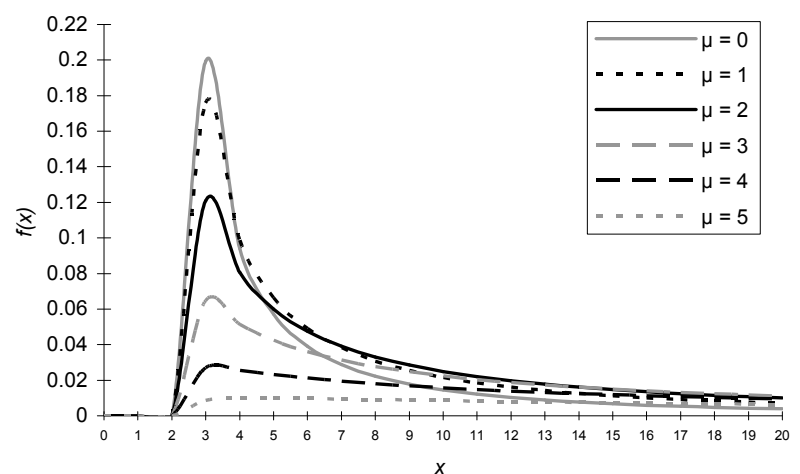

Figure 1. Probability density function of three-parametric lognormal distribution for the values of parameters $\sigma=2\left(\sigma^{2}=4\right) ; \theta=2$. [Source: Own research] 


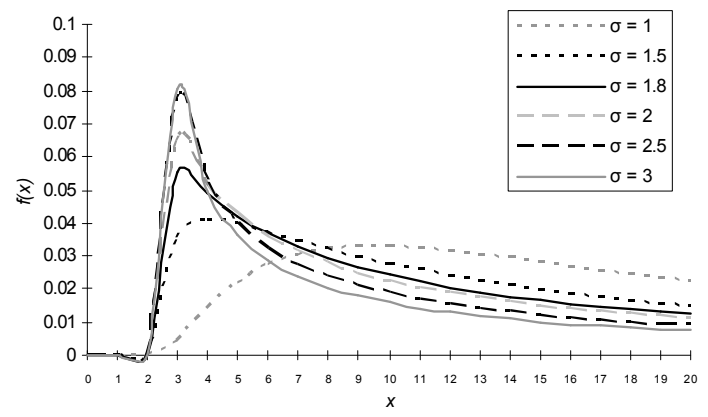

Figure 2. Probability density function of three-parametric lognormal distribution for the values of parameters $\mu=3 ; \theta=2$

[Source: Own research]

We obtain the expressions for the expected value and variance of random variable $X$ having three-parametric lognormal distribution from (57) and (58)

$$
\begin{gathered}
E(X)=\theta+\exp \left(\mu+\frac{\sigma^{2}}{2}\right), \\
D(X)=\exp \left(2 \mu+\sigma^{2}\right) \cdot\left[\exp \left(\sigma^{2}\right)-1\right]=\exp (2 \mu) \cdot \omega \cdot(\omega-1) .
\end{gathered}
$$

The expression for median

$$
\operatorname{Median}(X)=\theta+\exp (\mu)
$$

comes from the expression for $100 \cdot \mathrm{P} \%$ quantile of this distribution

$$
x_{P}=\theta+\exp \left(\mu+\sigma \cdot u_{P}\right)
$$

Three-parametric lognormal distribution is unimodal with one mode

$$
\operatorname{Mode}(X)=\theta+\exp \left(\mu-\sigma^{2}\right)=\theta+\frac{\exp (\mu)}{\omega}
$$

The relationship between the expected value, median and mode follows from the equations (61), (63) and (65)

$$
E(X)>\operatorname{Median}(X)>\operatorname{Mode}(X),
$$

which is typical just for positively skewed distribution.

The coefficient of variation of three-parametric lognormal distribution is a function of all three parameters $\mu, \sigma^{2}$ and $\theta$ of this distribution

$$
V(X)=\frac{\exp \left(\mu+\frac{\sigma^{2}}{2}\right) \sqrt{\exp \left(\sigma^{2}\right)-1}}{\theta+\exp \left(\mu+\frac{\sigma^{2}}{2}\right)}=\frac{\exp \left(\mu+\frac{\sigma^{2}}{2}\right) \sqrt{\omega-1}}{\theta+\exp \left(\mu+\frac{\sigma^{2}}{2}\right)} .
$$

Gini's coefficient of three-parametric lognormal distribution depends on all three parameters $\mu, \sigma^{2}$ and $\theta$ of this distribution, too

$$
G=\frac{\exp \left(\mu+\frac{\sigma^{2}}{2}\right) \cdot \operatorname{erf}\left(\frac{\sigma}{2}\right)}{\theta+\exp \left(\mu+\frac{\sigma^{2}}{2}\right)} .
$$

Moment measurement of skewness and kurtosis depend on a single parameter $\sigma^{2}$

$$
\begin{gathered}
\beta_{1}=\sqrt{\exp \left(\sigma^{2}\right)-1} \cdot\left[\exp \left(\sigma^{2}\right)+2\right]=\sqrt{\omega-1} \cdot(\omega+2), \\
\beta_{2}=\left[\exp \left(4 \sigma^{2}\right)+2 \exp \left(3 \sigma^{2}\right)+3 \exp \left(2 \sigma^{2}\right)-3\right]=\left(\omega^{4}+2 \omega^{3}+3 \omega^{2}-3\right) .
\end{gathered}
$$

\subsection{Four-Parametric Lognormal Curves}

Random variable $X$ has four parametric lognormal distribution with parameters $\mu, \sigma 2, \theta$ a $\tau$, where $-\infty<\mu<\infty$, $\sigma 2>0,-\infty<\theta<\tau<\infty$, if its probability density function has the form

$$
f\left(x ; \mu, \sigma^{2}, \theta, \tau\right) \quad=\frac{(\tau-\theta)}{\sigma \cdot(x-\theta) \cdot(\tau-x) \cdot \sqrt{2 \pi}} \cdot \exp \left[-\frac{\left(\ln \frac{x-\theta}{\tau-x}-\mu\right)^{2}}{2 \sigma^{2}}\right],
$$

$=0$,

Lognormal distribution with parameters $\mu, \sigma^{2}, \theta$ a $\tau$ is marked $\operatorname{LN}\left(\mu, \sigma^{2}, \theta, \tau\right)$. The probability density function of four-parametric lognormal distribution can have very different shapes depending on the values of the parameters of the distribution, see Figures 3-5. Distribution may be also bimodal for $\sigma^{2}>2$ and $|\mu|<\sigma^{2} \cdot \sqrt{\left(1-2 / \sigma^{2}\right)}-2 \tanh ^{-1} \sqrt{\left(1-2 / \sigma^{2}\right)} . \quad$ Probability density function of four-parametric lognormal distribution is often presented in the form

$$
f(x ; \gamma, \delta, \theta, \tau) \quad=\frac{\delta \cdot(\tau-\theta)}{(x-\theta) \cdot(\tau-x) \cdot \sqrt{2 \pi}} \cdot \exp \left[-\frac{1}{2}\left(\gamma+\delta \cdot \ln \frac{x-\theta}{\tau-x}\right)^{2}\right]
$$

$$
=0 \text {, }
$$

where it is valid between the expressions for probability density function (71) and (72) $\mu=-\frac{\gamma}{\delta}$ and $\sigma=\frac{1}{\delta}$.

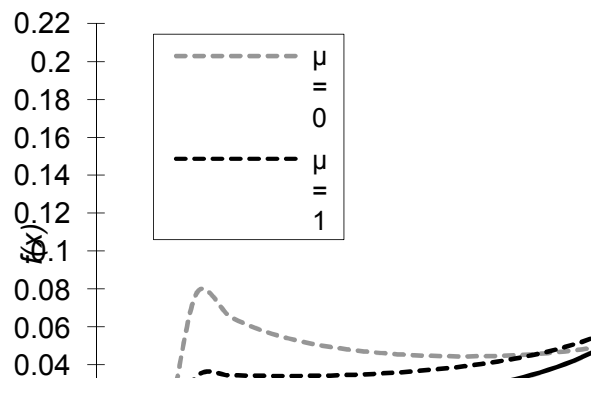

Figure 3. Probability density function of four-parametric lognormal distribution for the values of parameters $\sigma=2\left(\sigma^{2}=4\right) ; \theta=2 ; \tau=20$ [Source: Own research] 


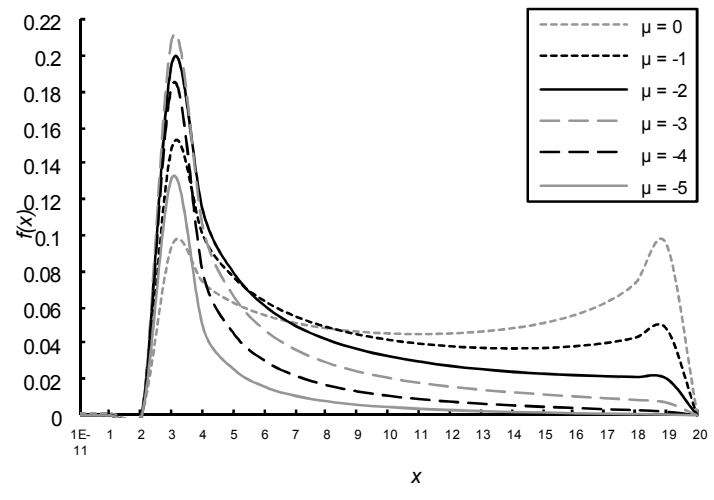

Figure 4. Probability density function of four-parametric lognormal distribution for the values of parameters $\sigma=2\left(\sigma^{2}=4\right) ; \theta=2 ; \tau=20$ [Source: Own research]

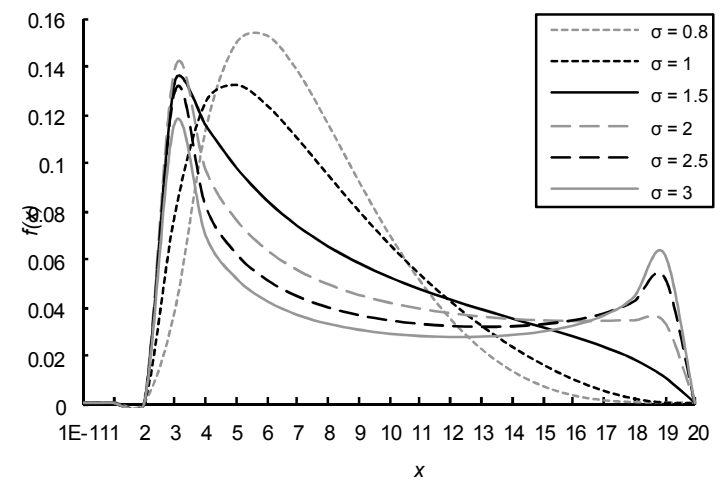

Figure 5. Probability density function of four-parametric lognormal distribution for the values of parameters $\mu=-1 ; \theta=2 ; \tau=20$ [Source: Own research]

If the random variable $X$ has four-parametric lognormal distribution $\operatorname{LN}\left(\mu, \sigma^{2}, \theta, \tau\right)$, then the random variable

$$
Y=\ln \frac{X-\theta}{\tau-X}
$$

has normal distribution $\mathrm{N}\left(\mu, \sigma^{2}\right)$ and the random variable

$$
U=\frac{\ln \frac{X-\theta}{\tau-X}-\mu}{\sigma}=\gamma+\delta \cdot \ln \frac{X-\theta}{\tau-X}
$$

has standardized normal distribution $\mathrm{N}(0 ; 1)$. Parameter $\mu$ is therefore the expected value of a random variable (73) and the parameter $\sigma^{2}$ is the variance of this random variable. The parameter $\theta$ is the beginning of the distribution (theoretical minimum) of a random variable $X$ and the parameter $\tau$ represents the end point of the distribution (theoretical maximum) of the random variable $X$.

More on the lognormal distribution is for example in [9] or [14]-[17].

\section{Methods of Point Parameter Estimation}

We focus here only on the parameter estimation of three-parametric lognormal distribution, which is the basic theoretical probability distribution of this research. Various methods of parametric estimation can be used for estimating the parameters of three-parametric lognormal distribution. There are for example the maximum likelihood method, moment method, quantile method, Kemsley's method, Cohen's method, L-moment method, TL-moment method, graphical method, etc. We focus on maximum likelihood method and on lesser-known methods of parametric estimation, i.e. Kemsley's method and Cohen's method.

\subsection{Maximum Likelihood Method}

Let the random sample of the sample size $n$ comes from three-parametric lognormal distribution with probability density function (52) or (53). Then the likelihood function have the form

$$
\begin{gathered}
L\left(\boldsymbol{x} ; \mu, \sigma^{2}, \theta\right)=\prod_{i=1}^{n} f\left(x_{i} ; \mu, \sigma^{2}, \theta\right)= \\
=\frac{1}{\left(\sigma^{2}\right)^{n / 2} \cdot(2 \pi)^{n / 2} \cdot \prod_{i=1}^{n}\left(x_{i}-\theta\right)} \cdot \exp \left\{\sum_{i=1}^{n}-\frac{\left[\ln \left(x_{i}-\theta\right)-\mu\right]^{2}}{2 \sigma^{2}}\right\} .
\end{gathered}
$$

We determine the logarithm of the likelihood function

$$
\ln L\left(\boldsymbol{x} ; \mu, \sigma^{2}, \theta\right)=\sum_{i=1}^{n}-\frac{\left[\ln \left(x_{i}-\theta\right)-\mu\right]^{2}}{2 \sigma^{2}}-\frac{n}{2} \cdot \ln \sigma^{2}-\frac{n}{2} \cdot \ln (2 \pi)-\sum_{i=1}^{n} \ln \left(x_{i}-\theta\right) .
$$

We put in the equality to zero the first partial derivation of the logarithm of the likelihood function according to $\mu$ and according to $\sigma^{2}$ by

$$
\begin{gathered}
\frac{\partial \ln L\left(\boldsymbol{x} ; \mu, \sigma^{2}, \theta\right)}{\partial \mu}=\frac{\sum_{i=1}^{n}\left[\ln \left(x_{i}-\theta\right)-\mu\right]}{\sigma^{2}}=0, \\
\frac{\partial \ln L\left(\boldsymbol{x} ; \mu, \sigma^{2}, \theta\right)}{\partial \sigma^{2}}=\frac{\sum_{i=1}^{n}\left[\ln \left(x_{i}-\theta\right)-\mu\right]^{2}}{2 \sigma^{4}}-\frac{n}{2 \sigma^{2}}=0 .
\end{gathered}
$$

We obtain maximum likelihood estimations of the parameters $\mu$ and $\sigma^{2}$ for the given parameter $\theta$ after treatment

$$
\hat{\mu}(\theta)=\frac{\sum_{i=1}^{n} \ln \left(x_{i}-\theta\right)}{n},
$$




$$
\hat{\sigma}^{2}(\theta)=\frac{\sum_{i=1}^{n}\left[\ln \left(x_{i}-\theta\right)-\hat{\mu}(\theta)\right]^{2}}{n} .
$$

If the value of the parameter $\theta$ in known, we get the maximum likelihood estimations of the remaining two parameters of three-parametric lognormal distribution using the expressions (79) and (80). However, if the value of the parameter $\theta$ is unknown, the problem is more complicated. It can be proved that if the parameter $\theta$ closes to $\min \left\{X_{1}, X_{2}, \ldots\right.$, $\left.X_{n}\right\}$, then the maximum likelihood approaches to infinity. The maximum likelihood method is also often combined with Cohen's method, where we put the smallest sample value to be equal to the $100 \cdot(n+1)^{-1}$-percentage quantile

$$
x_{\min }^{V}=\hat{\theta}+\exp \left(\hat{\mu}+\hat{\sigma} \cdot u_{(n+1)^{-1}}\right) .
$$

Equation (81) is then combined with a szstem of equations (79) and (80).

For solving of maximum likelihood equations (79) and (80) it is also possible to use $\theta$ satisfying the equation

$$
\sum_{i=1}^{n}\left(x_{i}-\hat{\theta}\right)+\frac{\sum_{i=1}^{n} \frac{z_{i}}{\left(x_{i}-\hat{\theta}\right)}}{\hat{\sigma}(\hat{\theta})}=0
$$

where

$$
z_{i}=\frac{\ln \left(x_{i}-\hat{\theta}\right)-\hat{\mu}(\hat{\theta})}{\hat{\sigma}(\hat{\theta})}
$$

where $\hat{\mu}(\hat{\theta})$ and $\hat{\sigma}(\hat{\theta})$ satisfy equations (79) and (80) with the parameter $\theta$ replaced by $\hat{\theta}$. We may also obtain the limits of variances

$$
\begin{gathered}
n \cdot D(\hat{\theta})=\frac{\sigma^{2} \cdot \exp (2 \mu)}{\omega \cdot\left[\omega \cdot\left(1+\sigma^{2}\right)-2 \sigma^{2}-1\right]}, \\
n \cdot D(\hat{\mu})=\frac{\sigma^{2} \cdot\left[\omega \cdot\left(1+\sigma^{2}\right)-2 \sigma^{2}\right]}{\omega \cdot\left(1+\sigma^{2}\right)-2 \sigma^{2}-1}, \\
n \cdot D(\hat{\sigma})=\frac{\sigma^{2} \cdot\left[\omega \cdot\left(1+\sigma^{2}\right)-1\right]}{\omega \cdot\left(1+\sigma^{2}\right)-2 \sigma^{2}-1} .
\end{gathered}
$$

Especially difficulties related with the use of the equations (79), (80) and (82) lead us to think about other methods.

\subsection{Kemsley's Method}

Kemsley used the estimation method, which is a combination of moment and quantile methods of parametric estimation. This method of parametric estimation put into equality the sample quantiles $x_{P 1}^{\mathrm{V}}$ and $x_{1-P 1}^{\mathrm{V}}$ and the corresponding theoretical quantiles of the probability distribution. We get the last equation so that we put sample average equal to the expected value of the probability distribution ("K" means Kemsley's estimation)

$$
\begin{gathered}
x_{P 1}^{\mathrm{V}}=\theta^{\mathrm{K}}+\exp \left(\mu^{\mathrm{K}}+\sigma^{\mathrm{K}} \cdot u_{P 1}\right), \\
\bar{x}=\theta^{\mathrm{K}}+\exp \left(\mu^{\mathrm{K}}+\frac{\sigma^{2^{\mathrm{K}}}}{2}\right), \\
x_{1-P 1}^{\mathrm{V}}=\theta^{\mathrm{K}}+\exp \left(\mu^{\mathrm{K}}-\sigma^{\mathrm{K}} \cdot u_{P 1}\right) .
\end{gathered}
$$

Now we solve a similar system of equations as in the case of quantile method of parameter estimation and

$$
f\left(\sigma^{2^{\mathrm{K}}}\right)=\frac{\exp \left(\frac{\sigma^{2^{\mathrm{K}}}}{2}\right)-\exp \left(\sigma^{\mathrm{K}} \cdot u_{P_{1}}\right)}{\exp \left(-\sigma^{\mathrm{K}} \cdot u_{P 1}\right)-\exp \left(\frac{\sigma^{2^{\mathrm{K}}}}{2}\right)}=\frac{\bar{x}-x_{P_{1}}^{\mathrm{V}}}{x_{\left(1-P_{1}\right)}^{\mathrm{V}}-\bar{x}} .
$$

The proposal for the solution of equation $\sigma^{2}{ }^{\mathrm{K}}$ determines approximately using Figure 6.

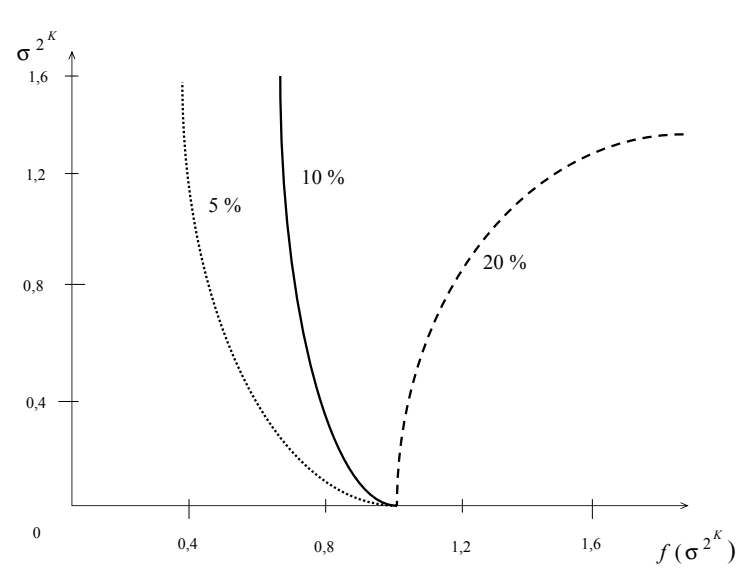

Figure 6. Graph $\sigma^{2}{ }^{\mathrm{K}}$ for Kemsley's method of parametric estimation for $p_{1}=0,05 ; 0,10$ and 0,20 .

[Source: Own research]

Then we obtain the values of the remaining two parameters using the expressions

$$
\begin{gathered}
\mu^{\mathrm{K}}=\ln \left(x_{P_{1}}^{\mathrm{V}}-\bar{x}\right)-\ln \left[\exp \left(\sigma^{\mathrm{K}} \cdot u_{P_{1}}\right)-\exp \left(\frac{\sigma^{2^{\mathrm{K}}}}{2}\right)\right], \\
\theta^{\mathrm{K}}=x_{P_{1}}^{\mathrm{V}}-\exp \left(\mu^{\mathrm{K}}+\sigma^{\mathrm{K}} \cdot u_{P 1}\right) .
\end{gathered}
$$




\subsection{Cohen's Method of the Smallest Sample Value}

It is known that parameter $\theta$ determines the beginning of three-parametric lognormal distribution. In this case, an appropriate estimation would be a function of the smallest sample value. This method constitutes an alternative to the method of maximum likelihood. This keeps the equations (79) and (80) and needed the third equation is based on the smallest sample value $x_{\min }$. If the value $x_{\min }$ is contained $n_{\min }$-times in the sample, then the sample quantile of order $n_{\text {min }} / n$ in the third equation is putted into equality to the corresponding theoretical quantile of the distribution. Thus, Cohen's method represents a combination of maximum likelihood method and the quantile method. We can get the parameter estimations obtained by Cohen's method with the system of equations ("CC" means Cohen's estimation)

$$
\begin{gathered}
\mu^{\mathrm{C}}=\frac{\sum_{i=1}^{n} \ln \left(x_{i}-\theta^{\mathrm{C}}\right)}{n}, \\
\sigma^{2^{\mathrm{C}}}=\frac{\sum_{i=1}^{n}\left[\ln \left(x_{i}-\theta^{\mathrm{C}}\right)-\mu^{\mathrm{C}}\right]^{2}}{n}, \\
\theta^{\mathrm{C}}={ }_{x_{n \min / n}}-\exp \left(\mu^{\mathrm{C}}+\sigma^{\mathrm{C}} \cdot u_{n \min / n}\right) .
\end{gathered}
$$

\section{Appropriateness of the Model}

It is also necessary to assess the suitability of constructed model or choose a model from several alternatives, which is made by some criterion, which can be a sum of absolute deviations of the observed and theoretical frequencies for all intervals

$$
S=\sum_{i=1}^{k}\left|n_{i}-n \pi_{i}\right|
$$

or known criterion $\chi^{2}$

$$
\chi^{2}=\sum_{i=1}^{k} \frac{\left(n_{i}-n \pi_{i}\right)^{2}}{n \pi_{i}},
$$

where ni are the observed frequencies in individual intervals, $\pi \mathrm{i}$ are the theoretical probabilities of membership of statistical unit into the $\mathrm{i}$-th interval, $\mathrm{n}$ is the total sample size of corresponding statistical file, $\mathrm{n} \cdot \pi \mathrm{i}$ are the theoretical frequencies in individual intervals, $\mathrm{i}=1,2, \ldots, \mathrm{k}$, and $\mathrm{k}$ is the number of intervals.

The question of the appropriateness of the given curve for model of the distribution of wage is not entirely conventional mathematical-statistical problem in which we test the null hypothesis

$\mathrm{H}_{0}$ : The sample comes from the supposed theoretical distribution against the alternative hypothesis
$\mathrm{H}_{1}$ : non $\mathrm{H}_{0}$, because in goodness of fit tests in the case of wage distribution we meet frequently with the fact that we work with large sample sizes and therefore the tests would almost always lead to the rejection of the null hypothesis. This results not only from the fact that with such large sample sizes the power of the test is so high at the chosen significance level that the test uncovers all the slightest deviations of the actual wage distribution and a model, but it also results from the principle of construction of the test. But practically we are not interested in such small deviations, so only gross agreement of the model with reality is sufficient and we so called "borrow" the model (curve). Test criterion $\chi^{2}$ can be used in that direction only tentatively. When evaluating the suitability of the model we proceed to a large extent subjective and we rely on experience and logical analysis.

\section{Data Base}

The data base of the research consists in employees of the Czech Republic. There are a total set of all employees of the Czech Republic together and further the partial sets broken down by various demographic and socio-economic factors. Figures 7 and 8 provide information on the geographic position of the Czech Republic in Europe and look at the map of the Czech Republic. The researched variable is the gross monthly wage in CZK (nominal wage). Data come from the official website of the Czech Statistical Office. The data was in the form of interval frequency distribution, since the individual data is not currently available.

Researched period represents years 2003-2010. Employees of the Czech Republic were classified according to gender, job classification (CZ-ISCO), the classification of economic activities, age and educational attainment. Branch Classification of Economic Activities (OKEC) has been replaced by Classification of Economic Activities (CZ-NACE) during researched period. This fast therefore disrupts the continuity of the obtained time series during the analysis period.

The main classes of Job Classification CZ-ISCO form: Managers (code 1000); Professionals (code 2000); Technicians and Associate Professionals (code 3000); Clerical Support Workers (code 4000); Service and Sales Workers (code 5000); Skilled Agricultural, Forestry and Fishery Workers (code 6000); Craft and Related Trades Workers (code 7000); Plant and Machine Operators, and Aassemblers (code 8000); Elementary Occupations (code 9000).

The main classes of Branch Classification of Economic Activities - OKEC (years 2003-2008) are: A-B Agriculture, Fishing; C-E - Industry; F - Construction; G - Trade, Repairs; H - Hotels and Restaurants; I - Transport, Storage; J - Financial intermediation; K - Real Estate, Renting; L - Public Administration; M - Education; N health; O - Other Services.

The main classes of Classification of Economic 
Activities - CZ-NACE (years 2009-2010) represent: A Agriculture, Forestry and Fishing; B-E - Industry; F Construction; G-Wholesale and Retail Trade, Repair of Motor Vehicles and Motorcycles; $\mathrm{H}$ - Transportion and Storage; I - Accomodation and Food Service Activities; J - Information and Communication; K - Financial and Insurance Activities; L - Real Estate activities; M Professional, Scientific and Technical Activities; N Administrative and Support Service Activities; O - Public Administration and Defence, Compulsory Social Security; P - Education; Q - Human Health and Social Work Activities; R - Arts, Entertainment and Recreation; S Other Service Activities.

Classification by age include the following age intervals: to 19 years; from 20 to 24 years; from 25 to 29 years; from 30 to 34 years; from 35 to 39 years; from 40 to 44 years; from 45 to 49 years; from 50 to 54 years; from 55 to 59 years; from 60 to 64 years; from 65 years.

Classification according to educational attainment distinguishs the fpllowing five levels of educational attainment of the employee: Primary education; Apprenticeship; Secondary with GCE; Higher post-secondary schools; University.

Tables 4-9 provide information on the sample sizes of sample sets of employees of single researched wage distribution.

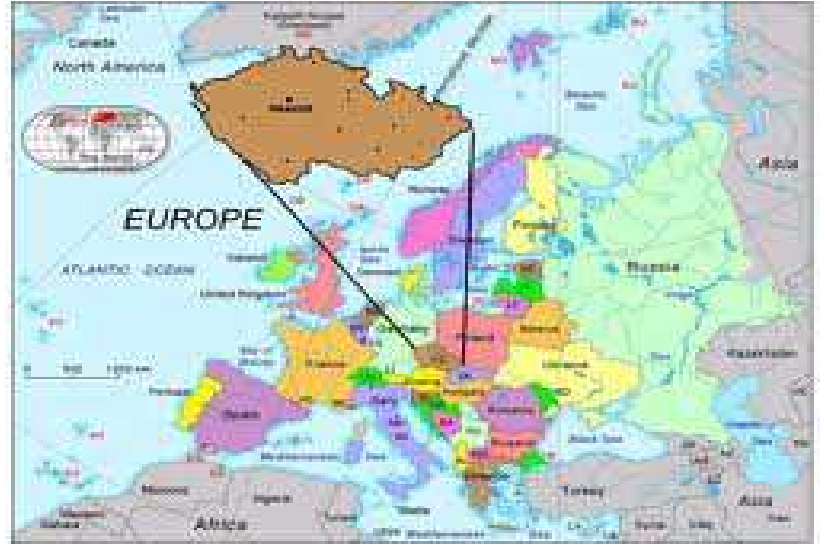

Figure 7. Position of the Czech Republic in Europe.

[Source: https://www.google.cz]

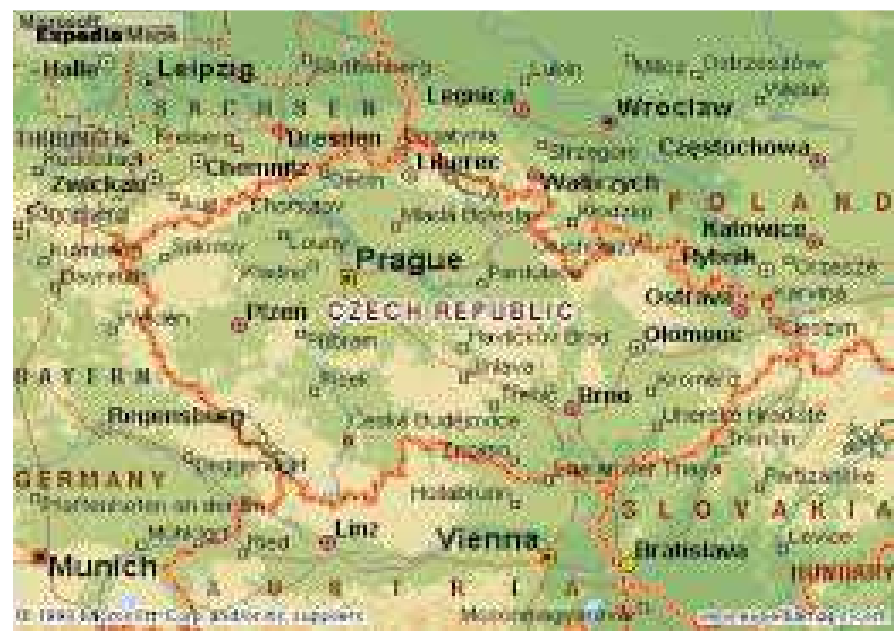

Figure 8. Map of the Czech Republic

[Source: https://www.google.cz]

Table 4. Sample sizes of wage distribution by gender.

\begin{tabular}{|c|c|c|c|c|c|c|c|c|}
\hline \multirow{2}{*}{ Gender } & \multicolumn{8}{|c|}{ Year } \\
\hline & 2003 & 2004 & 2005 & 2006 & 2007 & 2008 & 2009 & 2010 \\
\hline Total & $1,018,934$ & $1,404,496$ & $1,515,527$ & $1,614,372$ & $1,673,498$ & $1,711,811$ & $1,651,506$ & $1,662,829$ \\
\hline Men & 559,863 & 711,551 & 769,802 & 813,821 & 858,656 & 875,139 & 846,028 & 850,788 \\
\hline Women & 459,071 & 692,945 & 745,725 & 800,551 & 814,842 & 836,672 & 805,478 & 812,041 \\
\hline
\end{tabular}

Source: http://www.czso.cz

Table 5. Sample sizes of wage distribution by Job Classification CZ-ISCO.

\begin{tabular}{|c|c|c|c|c|c|c|c|c|}
\hline \multirow{2}{*}{ CZ-ISCO code } & \multicolumn{8}{|c|}{ Year } \\
\hline & 2003 & 2004 & 2005 & 2006 & 2007 & 2008 & 2009 & 2010 \\
\hline 1000 & 60,300 & 84,264 & 91,302 & 96,382 & 104,516 & 107,599 & 109,281 & 110,155 \\
\hline 2000 & 109,779 & 241,959 & 248,320 & 270,252 & 273,497 & 285,880 & 289,894 & 295,775 \\
\hline 3000 & 250,639 & 355,319 & 383,730 & 402,651 & 402,553 & 413,067 & 399,798 & 401,402 \\
\hline 4000 & 77,565 & 95,552 & 101,920 & 111,470 & 118,124 & 122,083 & 123,784 & 125,778 \\
\hline 5000 & 63,685 & 95,247 & 108,172 & 122,661 & 128,053 & 134,127 & 134,560 & 134,370 \\
\hline 6000 & 9,912 & 10,697 & 11,417 & 10,098 & 8,859 & 7,877 & 7,630 & 7,250 \\
\hline 7000 & 193,715 & 211,356 & 226,527 & 232,399 & 243,246 & 243,390 & 221,308 & 225,420 \\
\hline 8000 & 192,378 & 214,229 & 240,057 & 258,177 & 282,001 & 284,634 & 260,355 & 256,472 \\
\hline 9000 & 60,961 & 95,873 & 104,082 & 110,282 & 112,649 & 113,154 & 104,896 & 106,207 \\
\hline
\end{tabular}

Source: http://www.czso.cz 
Table 6. Sample sizes of wage distribution by Branch Classification of Economic Activities OKEC.

\begin{tabular}{|c|c|c|c|c|c|c|c|c|}
\hline \multirow{2}{*}{ OKEČ } & \multicolumn{8}{|c|}{ Year } \\
\hline & 2003 & 2004 & 2005 & 2006 & 2007 & 2008 & 2009 & 2010 \\
\hline$A+B$ & 28,132 & 31,055 & 33,004 & 27,502 & 24,296 & 21,537 & - & - \\
\hline C-E & 431,534 & 479,817 & 522,097 & 554,783 & 600,924 & 603,951 & - & - \\
\hline $\mathrm{F}$ & 38,261 & 42,223 & 45,242 & 43,941 & 50,073 & 50,437 & - & - \\
\hline G & 52,070 & 63,221 & 74,232 & 93,353 & 111,944 & 120,464 & - & - \\
\hline $\mathrm{H}$ & 8,556 & 11,188 & 12,020 & 15,447 & 16,858 & 16,997 & - & - \\
\hline I & 161,895 & 157,881 & 142,185 & 141,819 & 143,612 & 144,536 & - & - \\
\hline J & 47,932 & 52,140 & 48,601 & 51,893 & 53,506 & 55,993 & - & - \\
\hline $\mathrm{K}$ & 35,911 & 43,758 & 49,080 & 59,836 & 67,604 & 79,003 & - & - \\
\hline $\mathrm{L}$ & 68,971 & 192,993 & 217,590 & 235,536 & 232,800 & 233,438 & - & - \\
\hline M & 33,508 & 173,477 & 183,277 & 189,068 & 187,325 & 188,730 & - & - \\
\hline $\mathrm{N}$ & 93,480 & 125,784 & 149,429 & 160,700 & 144,471 & 155,533 & - & - \\
\hline $\mathrm{O}$ & 18,684 & 30,959 & 38,770 & 40,494 & 40,085 & 41,192 & - & - \\
\hline
\end{tabular}

Source: http://www.czso.cz

Table 7. Sample sizes of wage distribution by Classification of Economic Activities CZ-NACE.

\begin{tabular}{|c|c|c|c|c|c|c|c|c|}
\hline \multirow{2}{*}{ CZ-NACE } & \multicolumn{8}{|c|}{ Year } \\
\hline & 2003 & 2004 & 2005 & 2006 & 2007 & 2008 & 2009 & 2010 \\
\hline $\mathrm{A}$ & - & - & - & - & - & - & 20,560 & 18,659 \\
\hline B-E & - & - & - & - & - & - & 558,904 & 560,299 \\
\hline $\mathrm{F}$ & - & - & - & - & - & - & 50,789 & 52,769 \\
\hline G & - & - & - & - & - & - & 125,373 & 130,348 \\
\hline $\mathrm{H}$ & - & - & - & - & - & - & 147,328 & 141,193 \\
\hline I & - & - & - & - & - & - & 17,132 & 16,673 \\
\hline $\mathrm{J}$ & - & - & - & - & - & - & 42,058 & 43,602 \\
\hline K & - & - & - & - & - & - & 57,149 & 57,715 \\
\hline $\mathrm{L}$ & - & - & - & - & - & - & 5,540 & 5,093 \\
\hline M & - & - & - & - & - & - & 20,922 & 22,978 \\
\hline $\mathrm{N}$ & - & - & - & - & - & - & 41,588 & 44,533 \\
\hline $\mathrm{O}$ & - & - & - & - & - & - & 208,606 & 212,765 \\
\hline $\mathrm{P}$ & - & - & - & - & - & - & 185,453 & 186,092 \\
\hline Q & - & - & - & - & - & - & 143,595 & 143,877 \\
\hline $\mathrm{R}$ & - & - & - & - & - & - & 23,756 & 23,033 \\
\hline S & - & - & - & - & - & - & 2,753 & 3,200 \\
\hline
\end{tabular}

Source: http://www.czso.cz

Table 8. Sample sizes of wage distribution by age.

\begin{tabular}{|c|c|c|c|c|c|c|c|c|}
\hline \multirow{2}{*}{$\begin{array}{l}\text { Age } \\
\text { (in years) }\end{array}$} & \multicolumn{8}{|c|}{ Year } \\
\hline & 2003 & 2004 & 2005 & 2006 & 2007 & 2008 & 2009 & 2010 \\
\hline-19 & 2,805 & 3,567 & 4,314 & 5,887 & 6,879 & 6,455 & 4,245 & 3,927 \\
\hline $20-24$ & 63,496 & 76,595 & 86,317 & 97,025 & 105,523 & 106,958 & 94,097 & 91,160 \\
\hline $25-29$ & 129,298 & 166,682 & 178,259 & 188,289 & 193,222 & 190,866 & 177,961 & 177,044 \\
\hline $30-34$ & 121,054 & 173,799 & 197,020 & 217,720 & 227,325 & 231,284 & 220,500 & 216,899 \\
\hline $35-39$ & 122,324 & 170,268 & 183,513 & 198,609 & 210,780 & 226,740 & 233,095 & 246,619 \\
\hline $40-44$ & 123,278 & 184,904 & 204,368 & 218,373 & 225,528 & 226,265 & 216,461 & 218,695 \\
\hline $45-49$ & 148,936 & 198,188 & 205,107 & 208,653 & 209,454 & 217,468 & 220,087 & 227,237 \\
\hline $50-54$ & 166,456 & 221,988 & 222,759 & 220,744 & 220,894 & 216,944 & 201,687 & 194,387 \\
\hline $55-59$ & 113,813 & 163,222 & 182,059 & 194,592 & 200,682 & 207,352 & 201,606 & 203,674 \\
\hline $60-64$ & 22,019 & 36,571 & 42,151 & 52,473 & 60,501 & 66,795 & 66,452 & 68,220 \\
\hline $65+$ & 5,455 & 8,712 & 9,660 & 12,007 & 12,710 & 14,684 & 15,315 & 14,967 \\
\hline
\end{tabular}

Source: http://www.czso.cz

Table 9. Sample sizes of wage distribution by educational attainment.

\begin{tabular}{|c|c|c|c|c|c|c|c|c|}
\hline \multirow[b]{2}{*}{ Education } & \multicolumn{8}{|c|}{ Year } \\
\hline & 2003 & 2004 & 2005 & 2006 & 2007 & 2008 & 2009 & 2010 \\
\hline Primary education & 95,112 & 119,480 & 125,972 & 129,027 & 135,399 & 137,190 & 120,254 & 116,383 \\
\hline Apprenticeship & 377,347 & 470,688 & 523,744 & 553,522 & 587,081 & 591,669 & 557,780 & 555,266 \\
\hline Secondary with GCE & 408,562 & 560,237 & 575,668 & 621,306 & 629,447 & 644,576 & 625,631 & 627,073 \\
\hline Higher post-secondary schools & 15,749 & 29,144 & 40,055 & 42,856 & 47,967 & 54,439 & 57,747 & 64,684 \\
\hline University & 122,164 & 224,947 & 250,088 & 267,661 & 273,604 & 283,937 & 290,094 & 299,423 \\
\hline
\end{tabular}

Source: http://www.czso.cz 


\section{Results and Discussion}

All calculations were made using the statistical program packages Statgraphics and SAS, spreadsheet Microsoft Excel and mathematical program $\mathrm{R}$.

Figures 9 and 10 provide an overview of the development of the annual growth rate of the level of gross monthly wage in the Czech Republic in the period and the overview of the development of the average annual inflation. Because the growth rate is calculated from the growth coefficient, which is the ratio of two consecutive values of the time series, we would have data for 2002 to calculate the growth rate for the year 2003. Since 2002 is not included in the analysis period, the growth rate for 2003 is not presented here. The impact of the global economic crisis on the development of the wage level in the Czech Republic and on the development of inflation is clearly evident from these figures. It is apparent from Figure 9 that the annual growth rate of middle gross monthly wage in the Czech Republic dropped to almost zero in 2009. It has increased slightly over the next year, but it is far below the values before crisis. It is plainly evident from Figure 10 that the average annual inflation rate fell sharply in 2009 , but it again slightly increase during the next year, too.

Figure 11 presents the dependence of the value of criterion (96) on the sample size. A similar situation exists in term of the criterion (97). This is the wage distribution broken down by classification of economic activities CZ-NACE, i.e. a total of 32 wage distributions of the years 2009-2010. This is only a chosen file of wage distribution for clear visibility, since we obtain similar results also in terms of all surveyed wage distribution. The linear dependence of the value of criterion $S$ on the sample size follows from Figure 11. Figure 12 presents the results of significance tests for the linear dependence. We can see from Figure 12 that both significance tests of regression coefficient are significant at $5 \%$, but even at $1 \%$ significance level (t-test and F-test of regression coefficient provide equivalent results form the linear dependence between two variables). Linear dependence of the value of criterion $S$ on sample size is therefore proved even at $1 \%$ significance level.

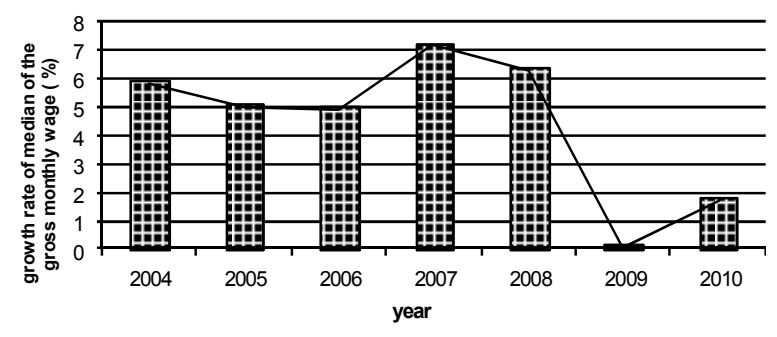

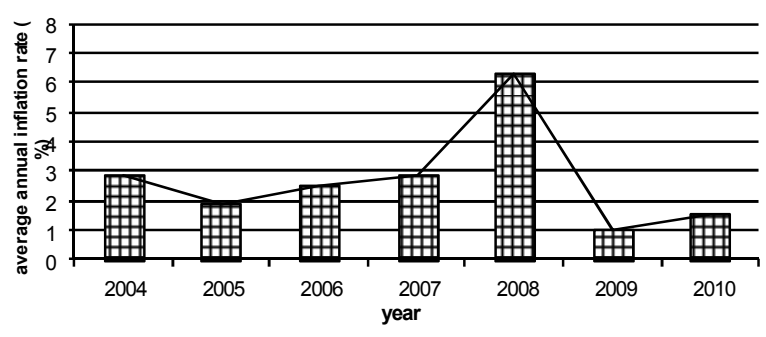

Figure 10. Average annual inflation rate in 2003-2010 (in \%) [Source: Own research]

The determination coefficient in Figure 12 says that $86.57 \%$ of the variability of criterion $S$ in given 32 wage distributions can be explained by the chosen regression line. The correlation coefficient in Figure 12 shows a very tight direct linear dependence of the criterion $S$ on sample size of wage distribution. Table 10 presents parameter estimations obtained using the various three methods of point parameter estimation and the value of criterion $S$ for the total wage distribution of the Czech Republic. This table describes approximately the research results of all 328 wage distribution. We obtained in total research that the method of TL-moments provided the most accurate results in almost all cases of wage distribution with minor exceptions, deviations occur mainly at both ends of the wage distribution due to the extreme open intervals of interval frequency distribution. In the results of Table 10 for total sets of wage distribution of the Czech Republic in 2003-2010 method of TL-moments always brings the most accurate results in terms of criterion $S$. In terms of research of all 328 wage distribution, method of L-moments brought the second most accurate results in more than in half of the cases. Deviations occur again especially at both ends of the distribution. In the results of Table 10 method of L-moments brought the second most accurate results in terms of all total sets of wage distribution of the Czech Republic in 2003-2010.

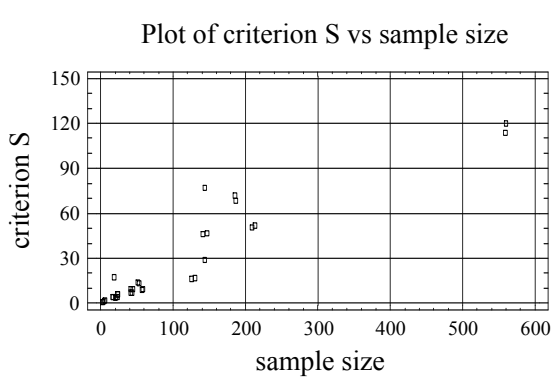

Figure 11. Dependence of the value of criterion $S$ (in 1,000) on sample size (in 1,000) - broken down by educational attainment, years 2003-2010 [Source: Own research]

Figure 9. Annual growth rate of the median of gross monthly wage in the Czech Republic in 2003-2010 (in \%t).

[Source: Own research] 


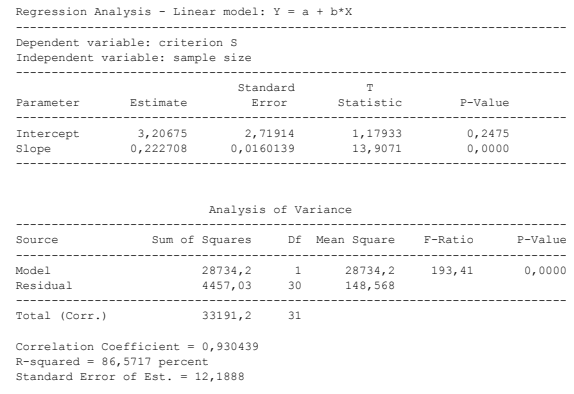

Figure 12. Dependence of the value of criterion $S$ (in 1,000) on sample size (in 1,000) - broken down by educational attainment, years 2003-20103) [Source: Own research]

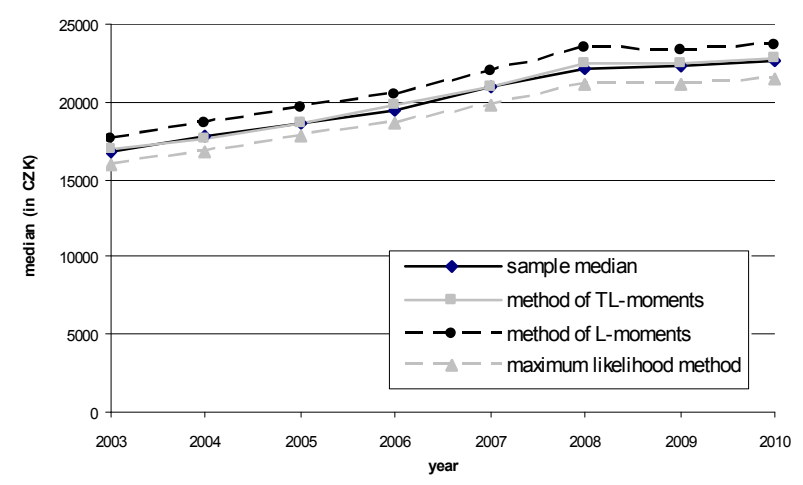

Figure 13. Development of sample and theoretical median of three-parametric lognormal curves with parameters estimated using three various methods of parameter estimation.

[Source: Own research]

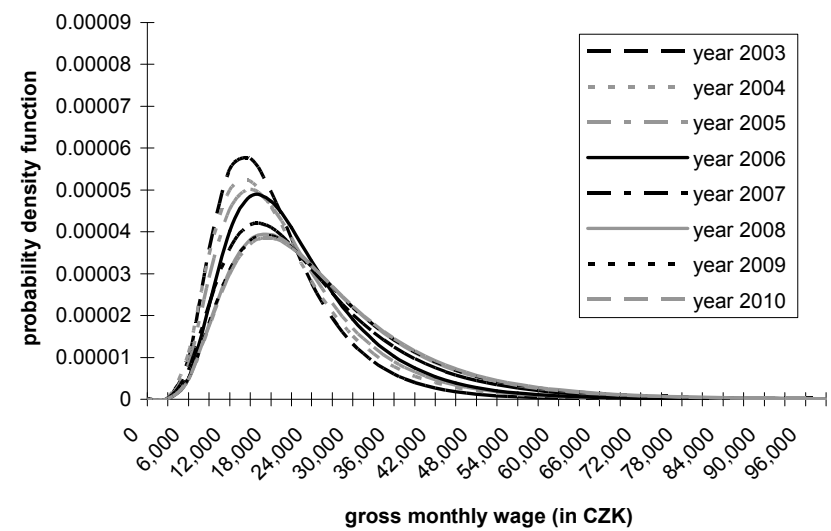

Figure 14. Development of probability density function of three-parametric lognormal curves with parameters estimated using the method of TL-moments.

[Source: Own research]

\footnotetext{
${ }^{3)}$ Output of the statistical program Statgraphics; decimal comma is used instead decimal point in this output
}

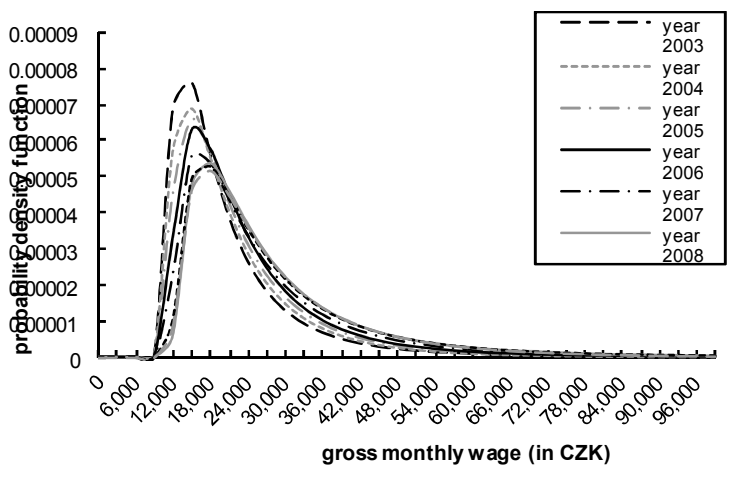

Figure 15. Development of probability density function of three-parametric lognormal curves with parameters estimated using the method of L-moments. [Source: Own research]

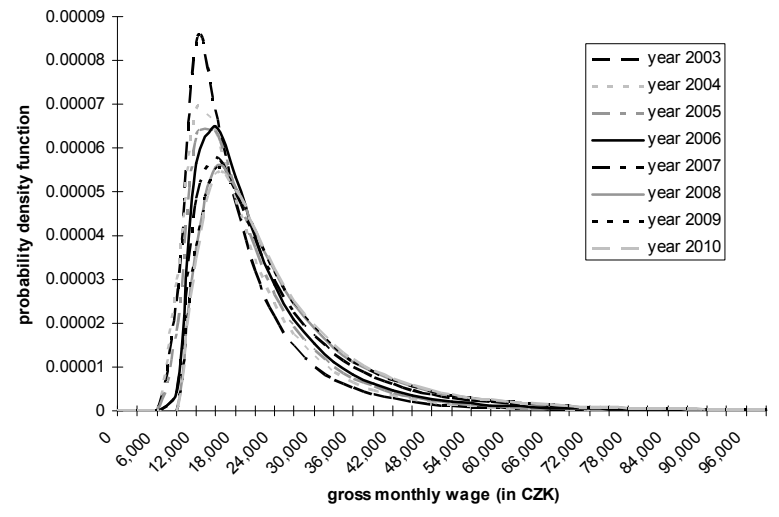

Figure 16. Development of probability density function of three-parametric lognormal curves with parameters estimated using the maximum likelihood method.

[Source: Own research]

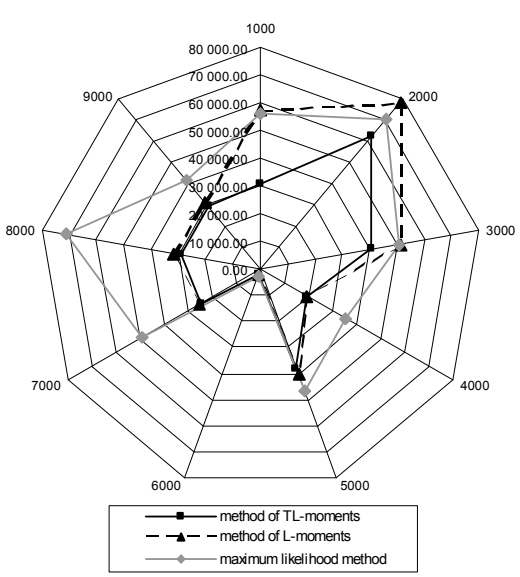

Figure 17. The values of S criterion for model three-parametric lognormal curves with parameters estimated by methods of point parameter estimation (year 2010, broken down by codes of job classification).

[Source: Own research] 


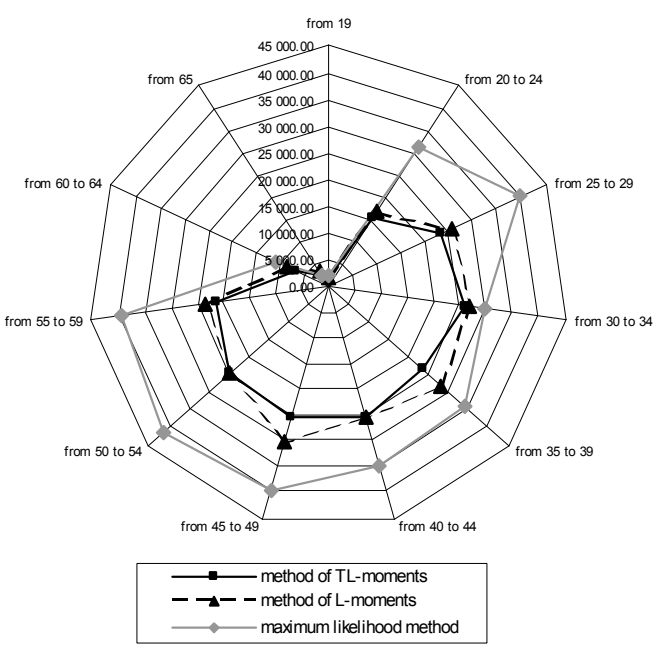

Figure 18. The values of S criterion for model three-parametric lognormal curves with parameters estimated by methods of point parameter estimation (year 2010, broken down by age intervals in years).

[Source: Own research]

Overall, maximum likelihood method was the third in most cases in terms of accuracy of the results obtained (in all cases in Table 10). Figure 13 also gives some idea of the accuracy of the researched methods of point parameter estimation. This figure shows the development of the sample median of gross monthly wage for the total set of all employees of the Czech Republic together in the period 2003-2010 and the development of corresponding theoretical median of model three-parametric lognormal curves with parameters estimated by three various methods of point parameter estimation. We can observe from this figure that the curve characterizing the course of theoretical median of three-parametric lognormal distribution with parameters estimated using the method of TL-moments adheres the most to the curve showing the development of the sample median. The other two curves articulating the development of the theoretical median of three-parametric lognormal curves with parameters estimated by method of L-moments and by maximum likelihood method are relatively remote from the course of sample median of wage distribution.

Table 10. Parameter estimations obtained using the various three methods of point parameter estimation and the value of criterion $S$ for the total wage distribution of the Czech Republic.

\begin{tabular}{|c|c|c|c|c|c|c|c|c|c|}
\hline \multirow{3}{*}{ Year } & \multicolumn{9}{|c|}{ Method } \\
\hline & \multicolumn{3}{|c|}{$\begin{array}{l}\text { Method of TL-moments } \\
\text { Parameter estimation }\end{array}$} & \multicolumn{3}{|c|}{$\begin{array}{l}\text { Method of L-moments } \\
\text { Parameter estimation }\end{array}$} & \multicolumn{3}{|c|}{$\begin{array}{c}\text { Maximum likelihood method in } \\
\text { combination with Cohen's method } \\
\text { Parameter estimation }\end{array}$} \\
\hline & $\mu$ & $\sigma^{2}$ & $\boldsymbol{\theta}$ & $\mu$ & $\sigma^{2}$ & $\theta$ & $\mu$ & $\sigma^{2}$ & $\boldsymbol{\theta}$ \\
\hline 2003 & 9.059747 & 0.630754 & $9,065.52$ & 9.017534 & 0.608369 & $7,664.46$ & 9.741305 & 0.197395 & 2.07 \\
\hline 2004 & 9.215324 & 0.581251 & $8,552.10$ & 9.241235 & 0.507676 & $6,541.16$ & 9.780008 & 0.232406 & 0.22 \\
\hline 2005 & 9.277248 & 0.573002 & $8,872.54$ & 9.283399 & 0.515290 & $6,977.45$ & 9.833604 & 0.228654 & 0.27 \\
\hline 2006 & 9.313803 & 0.577726 & $9,382.66$ & 9.283883 & 0.543225 & $7,868.21$ & 9.890594 & 0.210672 & 0.59 \\
\hline 2007 & 9.382135 & 0.680571 & $10,027.84$ & 9.387739 & 0.601135 & $7,902.64$ & 9.950263 & 0.268224 & 0.16 \\
\hline 2008 & 9.438936 & 0.688668 & $10,898.39$ & 9.423053 & 0.624340 & $8,754.64$ & 10.017433 & 0.264124 & 0.19 \\
\hline 2009 & 9.444217 & 0.703536 & $10,640.53$ & 9.431478 & 0.631013 & $8,684.51$ & 10.019787 & 0.269047 & 0.20 \\
\hline 2010 & 9.482060 & 0.681258 & $10,616.80$ & 9.453027 & 0.621057 & $8,746.20$ & 10.033810 & 0.269895 & 0.20 \\
\hline 2003 & & Criterion $S$ & & & Criterion $S$ & & & Criterion $S$ & \\
\hline 2004 & & $108,437.01$ & & & $133,320.79$ & & & $248,331.74$ & \\
\hline 2005 & & $146,509.34$ & & & $248,438.78$ & & & $281,541.41$ & \\
\hline 2006 & & $137,422.05$ & & & $231,978.79$ & & & $311,008.23$ & \\
\hline 2007 & & $149,144.68$ & & & $216,373.24$ & & & $325,055.67$ & \\
\hline 2008 & & $198,670.74$ & & & $366,202.87$ & & & $370,373.62$ & \\
\hline 2009 & & $206,698.93$ & & & $357,668.48$ & & & $391,346.02$ & \\
\hline 2010 & & $193,559.55$ & & & $335,999.20$ & & & $359,736.37$ & \\
\hline
\end{tabular}

Source: Own research

Figures 14-16 represents the development of probability density function of three-parametric lognormal curves with parameters estimated using the method of TL-moments, method of L-moments and maximum likelihood method. This is again a development of model distributions of the total wage distribution of the Czech Republic for all employees of the Czech Republic together in the period 2003-2010. We can see that the shapes of the lognormal curves with parameters estimated using the method of L-moments and maximum likelihood method (Figures 15 and 16) are similar mutually and they are very different from the shape of three-parametric lognormal curves with parameters estimated by the method of TL-moments (Figure 14).

Figure 17 shows the values of criterion $S$ of wage distributions broken down by job classification in 2010 and Figure 18 presents the same of wage distributions broken down by five-year age intervals in 2010. High accuracy of the method of TL-moments against the other two methods of point parameter estimation is evident from these two figures, too.

Other methods usable for processing of the economic data are presented for example in [18]-[21]. 


\section{Conclusions}

Alternative category of moment characteristics of probability distributions was introduced here. There are the characteristics in the form of L-moments and TL-moments. Accuracy of the methods of L-moments and TL-moments was compared with the accuracy of the maximum likelihood method using such criterion as the sum of all absolute deviations of the observed and theoretical frequencies for all intervals. Higher accuracy of the method of TL-moments due to the method of L-moments and to the maximum likelihood method was proved by studying of the set of 328 wage distribution. However, the advantages of the method of L-moments to the maximum likelihood method were demonstrated here, too. The values of $\chi 2$ criterion were also calculated for each wage distribution, but this test led always to the rejection of the null hypothesis about the supposed shape of the distribution due to the large sample sizes, which are typical for wage distribution. The dependence the value of criterion $\chi 2$ and the value of criterion of the sum of all absolute deviations of observed and theoretical frequencies on the sample size follows from the construction of the test. The linear dependence of the value of criterion of the sum of all absolute deviations of observed and theoretical frequencies on the sample size was proved even at $1 \%$ significance level.

\section{Acknowledgements}

This paper was subsidized by the funds of institutional support of a long-term conceptual advancement of science and research number IP400040 at the Faculty of Informatics and Statistics, University of Economics, Prague, Czech Republic.

\section{References}

[1] J. R. M. Hosking, "L-moments: Analysis and Estimation of Distributions Using Linear Combinations of Order Statistics," Journal of the Royal Statistical Society (Series B), 1990, Vol. 52, No. 1, pp. 105-124. ISSN 1467-9868.

[2] J. Kyselý and J. Picek, "Regional Growth Curves and Improved Design value Estimates of Extreme Precipitation Events in the Czech Republic," Climate research, 2007, Vol. 33, pp. 243-255. ISSN: 0936-577X.

[3] T. J. Ulrych, D. R.Velis, A. D. Woodbury and M. D. Sacchi, "L-moments and C-moments," Stoch. Environ. Res. Risk Asses, 2000, Vol. 14, pp. 50-68. ISSN 1436-3240.

[4] K. Adamowski, "Regional Analysis of Annual Maximum and Partial Duration Flood Data by Nonparametric and L-moment Methods," Journal of Hydrology, 2000, Vol. 229, pp. 219-231. ISSN 0022-1694.

[5] D. Bílková, "Modelling of Wage Distributions Using the Method of L-Moments," Paper presented at AMSE Applications of Mathematics and Statistics in Economy held on 25-28 August 2010, Demänovská Dolina, pp. 16-30. ISBN 978-80-89438-02-0.
[6] D. Bílková, "Use of the L-Moments Method in Modeling the Wage Distribution," Paper presented at Aplimat held on 01-04 February 2011, Bratislava, pp. 1471-1481. ISBN 978-80-89313-52-5.

[7] D. Bílková, "L-Moments and Their Use in Modeling the Distribution of Income and Wage," Paper presented at ISI held on 21-26 August 2011, Dublin, flashdisk, pp. 1-6.

[8] D. Bílková, "Modeling of Income and Wage Distribution Using the Method of L-Moments of Parameter Estimation," Paper presented at International Days of Statistics and Economics at VŠE held on 22-23 September 2011, Prague, pp. 1-10. ISBN 978-80-86175-72-0.

[9] D. Bílková, "Three-Parametric Lognormal Distribution and Estimating Its parameters Using the Method of L-Moments," Paper presented at RELIK - Reprodukce lidského kapitálu held on 05-06 December 2011, Prague, CD. ISBN 978-80-86175-75-1.

[10] D. Bílková, "Estimating Parameters of Lognormal Distribution Using the Method of L-Moments," Research Journal of Economics, Business and ICT, 2011, Vol. 4, No. 1, pp. 4-9. ISSN 2045-3345.

[11] D. Bílková, "Modelling of Wage and Income Distributions Using the Method of L-Moments," Journal of Mathematics and System Science, 2012, Vol. 2, No. 1, pp. 13-19. ISSN 2159-5291.

[12] D. Bílková and I. Malá, I. "Application of the L-Moment Method when Modelling the Income Distribution in the Czech Republic," Austrian Journal of Statistics, 2012, Vol. 41, No. 2, pp. 125-132. ISSN: 1026-597X.

[13] E. A. H. Elamir and A. H. Seheult, "Trimmed L-moments," Computational Statististics \& Data Analysis, 2003, Vol. 43, No. 3, pp. 299-314. ISSN: 0167-9473.

[14] D. Bílková, "Lognormal Distribution and Using L-Moment Method for Estimating Its Parameters," International Journal of Mathematical Models and Methods in Applied Sciences [online], 2012, Vol. 6, No. 1, pp. 30-44. ISSN 1998-0140. URL: http://www.naun.org/journals/m3as/17-079.pdf.

[15] D. Bílková, "Lognormal Distribution Parameter Estimating Using L-Moments," Journal of Mathematics and Technology, 2012, Vol. 3, No. 1, pp. 33-51. ISSN: 2078-0257.

[16] N. L. Johnson, S. Kotz and N. Balakrishnan, "Continuous Univariate Distributions", Wiley-Interscience, 1994. 756 p. ISBN 0-471-58495-9.

[17] C. Kleiber and S. Kotz , „Statistical Size Distributions in Economics and Actuarial Sciences", Wiley-Interscience, 2003. 332 p. ISBN 0-471-15064-9.

[18] T. Löster and J. Langhamrová, „Analysis of Long-Term Unemployment in the Czech Republic", Proceedings Conference International Days of Statististics and Economics at VŠE 2011, Prague, Czech Republic, 22.09.2011-23.09.2011, pp. 228-234, CD. ISBN 978-80-86175-72-0.

[19] L. Marek, "Wage development in the Czech Republic for the past 16 years," Paper presented at AIESA - Budovanie společnosti založenej na vedomostiach held on 10-11 November 2011, Bratislava, pp. 1-7. ISBN 978-80-225-3312-6. 
[20] L. Marek and M. Vrabec, "Mixture Normal Density Functions as a Model Wage Distribution," Paper presented at Economic, Marketing and Management held on 19-20 January 2013, Dubai, pp. 69-74. ISBN 978-981-07-5039-8. ISSN 2010-4626.
[21] M. H. Atyeh and W. Al-Rashed, „Testing the Existence of Integration; Kuwait and Jordan Financial Markets," International Journal of Economics, Finance and Management Sciences, 2013, Vol. 1 No. 2, pp. 89-94. doi: 10.11648/j.ijefm.20130102.14 